\title{
Extremal solutions to Liouville-Gelfand type elliptic problems with nonlinear Neumann boundary conditions
}

\author{
Futoshi Takahashi
}

\begin{tabular}{|c|l|}
\hline Citation & OCAMI Preprint Series \\
\hline Issue Date & 2013 \\
\hline Type & Preprint \\
\hline Textversion & Author \\
\hline Rights & $\begin{array}{l}\text { C } 2015 \text { World Scientific Publishing Company. For personal use only. No other } \\
\text { uses without permission. }\end{array}$ \\
\hline Relation & $\begin{array}{l}\text { Preprint of an article submitted for consideration in Communications in } \\
\text { Contemporary Mathematics } @ 2015 \text { World Scientific Publishing Company. } \\
\text { https://www.worldscientific.com/worldscinet/ccm }\end{array}$ \\
\hline Is version of & $\underline{\text { https://doi.org/10.1142/S0219199714500163 }}$ \\
\hline
\end{tabular}

From: Osaka City University Advanced Mathematical Institute http://www.sci.osaka-cu.ac.jp/OCAMI/publication/preprint/preprint.html 


\title{
Extremal solutions to Liouville-Gelfand type elliptic problems with nonlinear Neumann boundary conditions
}

\author{
Futoshi Takahashi \\ Department of Mathematics, Osaka City University \\ \& Osaka City University Advanced Mathematical Institute \\ Sumiyoshi-ku, Osaka, 558-8585, Japan \\ Tel: $(+81)(0) 6-6605-2508$ \\ E-mail: futoshi@sci.osaka-cu.ac.jp
}

Abstract. Consider the Liouville-Gelfand type problems with nonlinear Neumann boundary conditions

$$
\begin{cases}-\Delta u+u=0 & \text { in } \Omega \\ \frac{\partial u}{\partial \nu}=\lambda f(u) & \text { on } \partial \Omega,\end{cases}
$$

where $\Omega \subset \mathbb{R}^{N}, N \geq 2$, is a smooth bounded domain, $f:[0,+\infty) \rightarrow(0,+\infty)$ is a smooth, strictly positive, convex, increasing function with superlinear at $+\infty$, and $\lambda>0$ is a parameter. It is known that there exists an extremal parameter $\lambda^{*}>0$ such that a classical minimal solution exists for $\lambda<\lambda^{*}$, and there is no solution for $\lambda>\lambda^{*}$. In this paper, we prove several properties of extremal solutions $u^{*}$ corresponding to $\lambda=\lambda^{*}$, such as regularity, uniqueness, and the existence of weak eigenfunctions associated to the linearized extremal problem.

Keywords: Extremal solutions, Weak solutions, Nonlinear Neumann boundary conditions.

2010 Mathematics Subject Classifications: 35J20, 35J25. 35J60.

\section{Introduction}

Let $\Omega \subset \mathbb{R}^{N}(N \geq 2)$ be a smooth bounded domain and let $\nu$ denote the unit outer normal to $\partial \Omega$. Consider the Liouville-Gelfand type problems with 
nonlinear Neumann boundary conditions

$$
\begin{cases}-\Delta u+u=0 & \text { in } \Omega, \\ \frac{\partial u}{\partial \nu}=\lambda f(u) & \text { on } \partial \Omega,\end{cases}
$$

where $\lambda>0$ is a parameter. Throughout the paper, the nonlinearity $f$ : $[0,+\infty) \rightarrow(0,+\infty)$ is assumed to satisfy

$$
\begin{aligned}
& f \in C^{1}([0,+\infty)), f(0)>0, \text { convex, increasing, } \\
& \lim _{t \rightarrow+\infty} \frac{f(t)}{t}=+\infty
\end{aligned}
$$

Then maximum principle implies that solutions are positive on $\bar{\Omega}$.

Problem (1.1) may be considered as a variant of the well-studied problem

$$
\begin{cases}-\Delta u=\lambda f(u) & \text { in } \Omega, \\ u=0 & \text { on } \partial \Omega,\end{cases}
$$

where $\lambda>0$ and $f$ is assumed to satisfy (1.2), (1.3). For the problem (1.4), the notion of suitable weak solutions, the uniqueness and the regularity of extremal solutions, and the existence of the weak eigenfunction corresponding to zero eigenvalue of the linearized problem around the weak extremal solution, have been studied so far, see [3], [6], [13], [15], [7], and the references therein. Main purpose of this paper is to establish several facts for the problem (1.1), known to be true for (1.4). For other type of variants of the problem (1.4), see [4], [11].

Now, it is classic that the following proposition holds for the problem (1.1). The proof will be obtained by a slight modification of that of the similar proposition for the problem (1.4), see [9], [14], [10], [12], so we omit it.

\section{Proposition 1 Define}

$$
\lambda^{*}=\sup \left\{\lambda>0:(1.1)_{\lambda} \text { admits a classical solution } \in C^{2}(\bar{\Omega})\right\} .
$$

Then we have $0<\lambda^{*}<\infty$ and

(i) For every $\lambda \in\left(0, \lambda^{*}\right),(1.1)_{\lambda}$ has a positive, classical, minimal solution $u_{\lambda}$ in the sense that $u_{\lambda}(x) \leq u(x)(\forall x \in \bar{\Omega})$ for any other solution $u$ to 
$(1.1)_{\lambda}$. This is the unique strictly stable solution of $(1.1)_{\lambda}$, in the sense that

$$
\int_{\Omega}\left(|\nabla \varphi|^{2}+\varphi^{2}\right) d x>\lambda \int_{\partial \Omega} f^{\prime}\left(u_{\lambda}\right) \varphi^{2} d s_{x}
$$

holds for every $\varphi \in C^{1}(\bar{\Omega}), \varphi \not \equiv 0$.

(ii) The map $\lambda \mapsto u_{\lambda}(x)$ is continuous and increasing for any $x \in \bar{\Omega}$.

Motivated by the work by P. Quittner and W. Reichel [16], see also J. Dávila [10], we define the notion of weak solutions of (1.1) as follows.

Definition 2 ([16]) Let $L^{1}(\Omega \times \partial \Omega)$ denote the space of measurable functions $u$ on $\bar{\Omega}$ such that $\left.u\right|_{\Omega} \in L^{1}(\Omega)$ and $\left.u\right|_{\partial \Omega} \in L^{1}(\partial \Omega)$. $L^{1}(\Omega \times \partial \Omega)$ is a Banach space with the norm

$$
\|u\|_{L^{1}(\Omega \times \partial \Omega)}=\left\|\left.u\right|_{\Omega}\right\|_{L^{1}(\Omega)}+\left\|\left.u\right|_{\partial \Omega}\right\|_{L^{1}(\partial \Omega)} .
$$

As is remarked in [16], $\left.u\right|_{\Omega}$ and $\left.u\right|_{\partial \Omega}$ are not generally related with each other for $u \in L^{1}(\Omega \times \partial \Omega)$. The space $L^{1}(\Omega \times \partial \Omega)$ is isomorphic to $L^{1}(\Omega) \times$ $L^{1}(\partial \Omega)$. We use the notation $u=\left(u_{1}, u_{2}\right) \in L^{1}(\Omega) \times L^{1}(\partial \Omega)$ for $u \in L^{1}(\Omega \times$ $\partial \Omega)$, where $u_{1}=\left.u\right|_{\Omega}, u_{2}=\left.u\right|_{\partial \Omega}$.

Definition 3 Let $h \in L^{1}(\partial \Omega)$. We call a function $u=\left(u_{1}, u_{2}\right) \in L^{1}(\Omega) \times$ $L^{1}(\partial \Omega)$ is a weak solution to

$$
\begin{cases}-\Delta u+u=0 & \text { in } \Omega, \\ \frac{\partial u}{\partial \nu}=h & \text { on } \partial \Omega\end{cases}
$$

if it holds

$$
\int_{\Omega}(-\Delta \varphi+\varphi) u_{1} d x=\int_{\partial \Omega}\left(h \varphi-\frac{\partial \varphi}{\partial \nu} u_{2}\right) d s_{x}
$$

for any $\varphi \in C^{2}(\bar{\Omega})$. Also a function $u=\left(u_{1}, u_{2}\right) \in L^{1}(\Omega) \times L^{1}(\partial \Omega)$ is called a weak solution to $(1.1)_{\lambda}$ if $f\left(u_{2}\right) \in L^{1}(\partial \Omega)$ and

$$
\int_{\Omega}(-\Delta \varphi+\varphi) u_{1} d x=\int_{\partial \Omega}\left(\lambda f\left(u_{2}\right) \varphi-\frac{\partial \varphi}{\partial \nu} u_{2}\right) d s_{x}
$$

holds for any $\varphi \in C^{2}(\bar{\Omega})$. 
Remark 4 In some parts of the paper, admitting some ambiguity, we will identify $u_{1}$ or $u_{2}$ with $u$ for $u \in L^{1}(\Omega \times \partial \Omega)$.

Remark 5 If $u \in H^{1}(\Omega)$ is an energy solution to (1.1), that is, $f(u) \in$ $L^{1}(\partial \Omega)$ and

$$
\int_{\Omega}(\nabla u \cdot \nabla \varphi+u \varphi) d x=\int_{\partial \Omega} \lambda f(u) \varphi d s_{x}
$$

holds for any $\varphi \in C^{1}(\bar{\Omega})$, then $u$ is a weak solution in the sense of Definition 3 for $u_{1}=\left.u\right|_{\Omega}$ and $u_{2}=\left.u\right|_{\partial \Omega}$, here $\left.u\right|_{\partial \Omega} \in H^{1 / 2}(\partial \Omega)$ is the usual trace of $H^{1}$ function $u$ on $\partial \Omega$.

By Proposition 1, we may define a function

$$
u^{*}(x)=\lim _{\lambda \uparrow \lambda^{*}} u_{\lambda}(x), \quad x \in \bar{\Omega} .
$$

Then $u^{*}=\left(\left(u^{*}\right)_{1},\left(u^{*}\right)_{2}\right)=\left(\left.u^{*}\right|_{\Omega},\left.u^{*}\right|_{\partial \Omega}\right)$ becomes a weak solution of $(1.1)_{\lambda^{*}}$ in the sense of Definition 3. Indeed, let $\lambda_{1}>0$ denote the first eigenvalue of the Steklov type eigenvalue problem

$$
\begin{cases}-\Delta \varphi+\varphi=0 & \text { in } \Omega, \\ \frac{\partial \varphi}{\partial \nu}=\lambda \varphi & \text { on } \partial \Omega,\end{cases}
$$

and $\varphi_{1}$ the first eigenfunction. It is known that $\lambda_{1}$ is simple, isolated and $\varphi_{1}$ can be chosen positive (see [17]). Multiplying $\varphi_{1}$ to the equation of $u_{\lambda}$, we have

$$
\lambda_{1} \int_{\partial \Omega} u_{\lambda} \varphi_{1} d s_{x}=\lambda \int_{\partial \Omega} f\left(u_{\lambda}\right) \varphi_{1} d s_{x}
$$

Since $f$ satisfies the assumption (1.3), there exists a $C>0$ such that $f(t) \geq$ $\frac{4 \lambda_{1} t}{\lambda^{*}}-C$ for every $t>0$. Thus when $\lambda \in\left(\lambda^{*} / 2, \lambda^{*}\right)$, it holds

$$
\begin{aligned}
& \lambda_{1} \int_{\partial \Omega} u_{\lambda} \varphi_{1} d s_{x}=\lambda \int_{\partial \Omega} f\left(u_{\lambda}\right) \varphi_{1} d s_{x} \\
& \geq 2 \lambda_{1} \int_{\partial \Omega} u_{\lambda} \varphi_{1} d s_{x}-C,^{\prime} \quad\left(C^{\prime}=\frac{C \lambda^{*}}{2} \int_{\partial \Omega} \varphi_{1} d s_{x}\right) .
\end{aligned}
$$

Thus we have $\int_{\partial \Omega} u_{\lambda} \varphi_{1} d s_{x} \leq C$ and also $\int_{\partial \Omega} f\left(u_{\lambda}\right) \varphi_{1} d s_{x} \leq C$, where $C$ is independent of $\lambda \in\left(\lambda^{*} / 2, \lambda^{*}\right)$. Thus by Fatou's lemma and the fact $\left.\varphi_{1}\right|_{\partial \Omega} \geq$ 
$c_{0}>0$ for some $c_{0}>0$, we obtain that $u^{*} \in L^{1}(\partial \Omega)$ and $f\left(u^{*}\right) \in L^{1}(\partial \Omega)$. To see that $u^{*} \in L^{1}(\Omega)$, let $\zeta \in C^{2}(\Omega)$ be the solution of

$$
\begin{cases}-\Delta \zeta+\zeta=1 & \text { in } \Omega, \\ \frac{\partial \zeta}{\partial \nu}=0 & \text { on } \partial \Omega .\end{cases}
$$

Multiplying $\zeta$ to the equation of $u_{\lambda}$, we have

$$
\int_{\Omega} u_{\lambda} d x=\lambda \int_{\partial \Omega} f\left(u_{\lambda}\right) \zeta d s_{x} \leq C .
$$

Fatou's lemma again confirms that $u^{*} \in L^{1}(\Omega)$. Since $u_{\lambda}$ satisfies

$$
\int_{\Omega}(-\Delta \varphi+\varphi) u_{\lambda} d x=\int_{\partial \Omega}\left(\lambda f\left(u_{\lambda}\right) \varphi-\frac{\partial \varphi}{\partial \nu} u_{\lambda}\right) d s_{x}
$$

for any $\varphi \in C^{2}(\bar{\Omega})$, letting $\lambda \uparrow \lambda^{*}$ and using Lebesgue's dominated convergence theorem on $\Omega$ and $\partial \Omega$, we obtain that $u^{*}$ is a weak solution of $(1.1)_{\lambda^{*}}$. In the following, we call $u^{*}$ the extremal solution of (1.1).

The organization of the paper is as follows. In $\S 2$, we collect lemmas which will be used in the later sections. Several facts analogous to those established by Brezis, Cazenave, Martel and Ramiandrisoa [3] for (1.4) will be proved. In $\S 3$, we treat the regularity property of the extremal solution to (1.1), as in Nedev [15], see also Dávila [10]. In $§ 4$, similarly to the result by Martel [13], the uniqueness of the extremal solution among weak solutions will be proved. In $\S 5$, we study the existence of weak eigenfunctions corresponding to zero eigenvalue of the linearized eigenvalue problem around the extremal solution. Corresponding result for the problem (1.4) has been studied by Cábre and Martel [7].

\section{Preliminaries}

In this section, we prepare several useful lemmas in the sequel of the paper.

Lemma 6 Given $h \in L^{1}(\partial \Omega)$, there exists a unique weak solution $u=$ $\left(u_{1}, u_{2}\right) \in L^{1}(\Omega) \times L^{1}(\partial \Omega)$ to (1.7) in the sense of (1.8). Moreover, it holds

$$
\left\|u_{1}\right\|_{L^{1}(\Omega)}+\left\|u_{2}\right\|_{L^{1}(\partial \Omega)} \leq C\|h\|_{L^{1}(\partial \Omega)} .
$$

for some $C>0$ independent of $u$ and $h$. Also if $h \geq 0$ on $\partial \Omega$, then $u_{1}, u_{2} \geq 0$. 
Proof. We prove the uniqueness first. Let $u=\left(u_{1}, u_{2}\right), \tilde{u}=\left(\tilde{u}_{1}, \tilde{u}_{2}\right)$ be weak solutions. Then $w=\left(w_{1}, w_{2}\right), w_{1}=u_{1}-\tilde{u}_{1}, w_{2}=u_{2}-\tilde{u}_{2}$, satisfies

$$
\int_{\Omega}(-\Delta \varphi+\varphi) w_{1} d x=\int_{\partial \Omega}\left(-\frac{\partial \varphi}{\partial \nu}\right) w_{2} d s_{x}
$$

for any $\varphi \in C^{2}(\bar{\Omega})$. Given $\zeta \in C^{\infty}(\partial \Omega)$, let $\varphi \in C^{2}(\bar{\Omega})$ be the solution to

$$
\begin{cases}-\Delta \varphi+\varphi=0 & \text { in } \Omega, \\ \frac{\partial \varphi}{\partial \nu}=\zeta & \text { on } \partial \Omega .\end{cases}
$$

Then we have

$$
\int_{\partial \Omega} w_{2} \zeta d s_{x}=0
$$

for such $\zeta$, hence $w_{2}=0$ a.e. on $\partial \Omega$. Similarly, for given $\eta \in C_{0}^{\infty}(\Omega)$, let $\varphi \in C^{2}(\bar{\Omega})$ be the solution to

$$
\begin{cases}-\Delta \varphi+\varphi=\eta & \text { in } \Omega, \\ \frac{\partial \varphi}{\partial \nu}=0 & \text { on } \partial \Omega,\end{cases}
$$

then we have

$$
\int_{\Omega} w_{1} \eta d x=0
$$

and conclude $w_{1}=0$ a.e. on $\Omega$.

To prove the a priori estimate $(2.1)$, let $\varphi \in C^{2}(\Omega) \cap C(\bar{\Omega})$ be the solution to

$$
\begin{cases}-\Delta \varphi+\varphi=\operatorname{sgn}\left(u_{1}\right) \in L^{\infty}(\Omega) & \text { in } \Omega, \\ \frac{\partial \varphi}{\partial \nu}=0 & \text { on } \partial \Omega .\end{cases}
$$

By the definition of the weak solution, we have

$$
\int_{\Omega}\left|u_{1}\right| d x=\int_{\Omega}(-\Delta \varphi+\varphi) u_{1} d x=\int_{\partial \Omega} h \varphi d s_{x} \leq \max _{\partial \Omega}|\varphi|\|h\|_{L^{1}(\partial \Omega)} .
$$

By the maximum principle, we have $\max _{x \in \partial \Omega}|\varphi(x)| \leq 1$ and $\left\|u_{1}\right\|_{L^{1}(\Omega)} \leq$ $\|h\|_{L^{1}(\partial \Omega)}$. Similarly, we have $\left\|u_{2}\right\|_{L^{1}(\partial \Omega)} \leq C\|h\|_{L^{1}(\partial \Omega)}$ if we take $\varphi$ as the solution to

$$
\begin{cases}-\Delta \varphi+\varphi=0 & \text { in } \Omega, \\ \frac{\partial \varphi}{\partial \nu}=\operatorname{sgn}\left(u_{2}\right) \in L^{\infty}(\partial \Omega) & \text { on } \partial \Omega .\end{cases}
$$


Note that in this case $\max _{x \in \partial \Omega}|\varphi(x)| \leq \max _{x \in \partial \Omega}|\psi(x)|$, where $\psi$ is the solution to

$$
\begin{cases}-\Delta \psi+\psi=0 & \text { in } \Omega, \\ \frac{\partial \psi}{\partial \nu}=1 & \text { on } \partial \Omega .\end{cases}
$$

To prove the existence, put

$$
h_{m}(x)= \begin{cases}m & \text { if } h(x) \geq m \\ h(x) & \text { if }|h(x)| \leq m \\ -m & \text { if } h(x) \leq-m\end{cases}
$$

for $m \in \mathbb{N}$. Since $h_{m} \in L^{\infty}(\partial \Omega)$, there exists $u_{m} \in H^{1}(\Omega)$ such that

$$
\begin{cases}-\Delta u_{m}+u_{m}=0 & \text { in } \Omega, \\ \frac{\partial u_{m}}{\partial \nu}=h_{m} & \text { on } \partial \Omega .\end{cases}
$$

By the estimate (2.1), we have

$\left\|u_{m}-u_{n}\right\|_{L^{1}(\Omega)}+\left\|\left.u_{m}\right|_{\partial \Omega}-\left.u_{n}\right|_{\partial \Omega}\right\|_{L^{1}(\partial \Omega)} \leq C\left\|h_{m}-h_{n}\right\|_{L^{1}(\partial \Omega)}=o(1), \quad(m, n \rightarrow \infty)$.

Thus $\left\{u_{m}\right\}_{m \in \mathbb{N}}$ and $\left\{\left.u_{m}\right|_{\partial \Omega}\right\}_{m \in \mathbb{N}}$ are Cauchy sequences in $L^{1}(\Omega)$ and $L^{1}(\partial \Omega)$ respectively. Then there exist $u_{1} \in L^{1}(\Omega), u_{2} \in L^{1}(\partial \Omega)$ such that

$$
u_{m} \rightarrow u_{1} \quad \text { in } L^{1}(\Omega),\left.\quad u_{m}\right|_{\partial \Omega} \rightarrow u_{2} \quad \text { in } L^{1}(\partial \Omega) .
$$

Since $u_{m}$ satisfies

$$
\int_{\Omega}(-\Delta \varphi+\varphi) u_{m} d x=\int_{\partial \Omega}\left(h \varphi-\left.\frac{\partial \varphi}{\partial \nu} u_{m}\right|_{\partial \Omega}\right) d s_{x}
$$

for any $\varphi \in C^{2}(\bar{\Omega})$, we easily see that $\left(u_{1}, u_{2}\right) \in L^{1}(\Omega) \times L^{1}(\partial \Omega)$ is a weak solution of (1.7) by letting $m \rightarrow \infty$.

Lastly, if $h \geq 0$ on $\partial \Omega$, we have $h_{m} \geq 0$ and the maximum principle implies that $u_{m} \geq 0$ on $\bar{\Omega}$. Thus $u_{1} \geq 0$ on $\Omega$ and $u_{2} \geq 0$ on $\partial \Omega$.

Lemma 7 Let $h \in L^{1}(\partial \Omega)$ and let $u=\left(u_{1}, u_{2}\right) \in L^{1}(\Omega) \times L^{1}(\partial \Omega)$ be the weak solution to (1.7) in the sense of (1.8). Let $\Phi \in C^{2}(\mathbb{R})$ be concave, with $\Phi^{\prime}$ 
bounded and $\Phi(0)=0$. Then $v=\left(v_{1}, v_{2}\right)=\left(\Phi\left(u_{1}\right), \Phi\left(u_{2}\right)\right) \in L^{1}(\Omega) \times L^{1}(\partial \Omega)$ is a weak supersolution to

$$
\begin{cases}-\Delta v_{1}+v_{1}=0 & \text { in } \Omega, \\ \frac{\partial v_{2}}{\partial \nu}=\Phi^{\prime}\left(u_{2}\right) h & \text { on } \partial \Omega,\end{cases}
$$

in the sense that

$$
\int_{\Omega}(-\Delta \psi+\psi) v_{1} d x \geq \int_{\partial \Omega}\left\{\Phi^{\prime}\left(u_{2}\right) h \psi-\frac{\partial \psi}{\partial \nu} v_{2}\right\} d s_{x}
$$

for any $\psi \in C^{2}(\bar{\Omega}), \psi \geq 0$ on $\bar{\Omega}$.

Proof. For $h \in L^{1}(\partial \Omega)$ and $m \in \mathbb{N}$, define $h_{m}$ as before in (2.5) and let $u_{m} \in H^{1}(\Omega)$ be an energy solution of (2.6). By Lemma 6 , we know $u_{m} \rightarrow u_{1}$ in $L^{1}(\Omega),\left.u_{m}\right|_{\partial \Omega} \rightarrow u_{2}$ in $L^{1}(\partial \Omega)$, where $u=\left(u_{1}, u_{2}\right)$ is a weak solution of (1.7) in the sense of (1.8). From

$$
\begin{aligned}
& \left\|\Phi\left(u_{1}\right)-\Phi\left(u_{m}\right)\right\|_{L^{1}(\Omega)} \leq\left\|\Phi^{\prime}\right\|_{L^{\infty}(\mathbb{R})}\left\|u_{1}-u_{m}\right\|_{L^{1}(\Omega)}, \\
& \left\|\Phi\left(u_{2}\right)-\Phi\left(u_{m}\right)\right\|_{L^{1}(\partial \Omega)} \leq\left\|\Phi^{\prime}\right\|_{L^{\infty}(\mathbb{R})}\left\|u_{2}-u_{m}\right\|_{L^{1}(\partial \Omega)},
\end{aligned}
$$

we obtain $v=\left(v_{1}, v_{2}\right)=\left(\Phi\left(u_{1}\right), \Phi\left(u_{2}\right)\right) \in L^{1}(\Omega) \times L^{1}(\partial \Omega)$. Since $u_{m}$ satisfies

$$
\int_{\Omega}\left(\nabla \varphi \cdot \nabla u_{m}+\varphi u_{m}\right) d x=\int_{\partial \Omega} h_{m} \varphi d s_{x}
$$

for any $\varphi \in C^{2}(\bar{\Omega})$, by density argument, this holds true for any $\varphi \in H^{1}(\Omega)$. We take $\varphi=\Phi^{\prime}\left(u_{m}\right) \psi$ with $\psi \in C^{2}(\bar{\Omega}), \psi \geq 0$ on $\bar{\Omega}$, then we find that

$$
\int_{\Omega}\left\{\nabla\left(\Phi^{\prime}\left(u_{m}\right) \psi\right) \cdot \nabla u_{m}+\Phi^{\prime}\left(u_{m}\right) \psi u_{m}\right\} d x=\int_{\partial \Omega} h_{m} \Phi^{\prime}\left(u_{m}\right) \psi d s_{x} .
$$

Noting $\Phi^{\prime \prime} \leq 0$ and $\psi \geq 0$, we have

$$
\int_{\Omega}\left\{\nabla\left(\Phi\left(u_{m}\right)\right) \cdot \nabla \psi+\Phi^{\prime}\left(u_{m}\right) \psi u_{m}\right\} d x \geq \int_{\partial \Omega} h_{m} \Phi^{\prime}\left(u_{m}\right) \psi d s_{x} .
$$

Now, since $\Phi$ is concave with $\Phi(0)=0$, we have $\frac{\Phi\left(u_{m}\right)}{u_{m}} \geq \Phi^{\prime}\left(u_{m}\right)$. Therefore,

$$
\int_{\Omega}\left\{\nabla\left(\Phi\left(u_{m}\right)\right) \cdot \nabla \psi+\Phi\left(u_{m}\right) \psi\right\} d x \geq \int_{\partial \Omega} h_{m} \Phi^{\prime}\left(u_{m}\right) \psi d s_{x}
$$


holds for any $\psi \in C^{2}(\bar{\Omega}), \psi \geq 0$. Integration by parts leads to

$$
\int_{\Omega}(-\Delta \psi+\psi) \Phi\left(u_{m}\right) d x \geq \int_{\partial \Omega}\left\{h_{m} \Phi^{\prime}\left(u_{m}\right) \psi-\Phi\left(u_{m}\right) \frac{\partial \psi}{\partial \nu}\right\} d s_{x} .
$$

Passing to the limit with the estimates

$$
\begin{aligned}
& \left|\int_{\Omega}(-\Delta \psi+\psi)\left(\Phi\left(u_{1}\right)-\Phi\left(u_{m}\right)\right) d x\right| \\
& \leq\|-\Delta \psi+\psi\|_{L^{\infty}(\Omega)}\left\|\Phi^{\prime}\right\|_{L^{\infty}(\mathbb{R})}\left\|u_{m}-u_{1}\right\|_{L^{1}(\Omega)}=o(1), \\
& \left|\int_{\partial \Omega}\left(\Phi\left(u_{2}\right)-\Phi\left(u_{m}\right)\right) \frac{\partial \psi}{\partial \nu} d s_{x}\right| \leq\|\nabla \psi\|_{L^{\infty}(\partial \Omega)}\left\|\Phi^{\prime}\right\|_{L^{\infty}(\mathbb{R})}\left\|u_{m}-u_{2}\right\|_{L^{1}(\partial \Omega)}=o(1), \\
& \int_{\partial \Omega} h_{m} \Phi^{\prime}\left(u_{m}\right) \psi d s_{x} \rightarrow \int_{\partial \Omega} h \Phi^{\prime}\left(u_{2}\right) \psi d s_{x},
\end{aligned}
$$

we confirm that $v=\Phi(u)=\left(\Phi\left(u_{1}\right), \Phi\left(u_{2}\right)\right)$ is the desired weak supersolution. Note that $h_{m} \rightarrow h$ in $L^{1}(\partial \Omega)$ strongly. Thus the last estimate is assured by the Lebesgue dominated convergence theorem, since a.e. convergence along a subsequence and the estimate $\left|h_{m} \Phi^{\prime}\left(u_{m}\right) \psi\right| \leq\left\|\Phi^{\prime}\right\|_{L^{\infty}(\mathbb{R})}\|\psi\|_{L^{\infty}(\partial \Omega)}|h| \in$ $L^{1}(\partial \Omega)$ hold true.

Lemma 8 Assume $(1.1)_{\lambda}$ has a weak supersolution $\bar{w}=\left(\bar{w}_{1}, \bar{w}_{2}\right) \in L^{1}(\Omega) \times$ $L^{1}(\partial \Omega)$, in the sense that $f\left(\bar{w}_{2}\right) \in L^{1}(\partial \Omega)$ and

$$
\int_{\Omega}(-\Delta \varphi+\varphi) \bar{w}_{1} d x \geq \int_{\partial \Omega}\left\{\lambda f\left(\bar{w}_{2}\right) \varphi-\frac{\partial \varphi}{\partial \nu} \bar{w}_{2}\right\} d s_{x}
$$

for any $\varphi \in C^{2}(\bar{\Omega}), \varphi \geq 0$ on $\bar{\Omega}$. Then $(1.1)_{\lambda}$ has a weak solution $u=$ $\left(u_{1}, u_{2}\right) \in L^{1}(\Omega) \times L^{1}(\partial \Omega)$.

Proof. Proof consists of a standard monotone iteration argument in our context. Define $w^{(1)}=\left(w_{1}^{(1)}, w_{2}^{(1)}\right)=\bar{w}=\left(\bar{w}_{1}, \bar{w}_{2}\right) \in L^{1}(\Omega) \times L^{1}(\partial \Omega)$. By the definition, we have $f\left(w_{2}^{(1)}\right) \in L^{1}(\partial \Omega)$. Let $w^{(2)}=\left(w_{1}^{(2)}, w_{2}^{(2)}\right)$ be the unique weak solution of

$$
\begin{cases}-\Delta w_{1}^{(2)}+w_{1}^{(2)}=0 & \text { in } \Omega, \\ \frac{\partial w_{2}^{(2)}}{\partial \nu}=\lambda f\left(w_{2}^{(1)}\right) & \text { on } \partial \Omega\end{cases}
$$


obtained by Lemma 6 . Thus,

$$
\int_{\Omega}(-\Delta \varphi+\varphi)\left(w_{1}^{(1)}-w_{1}^{(2)}\right) d x \geq \int_{\partial \Omega} \frac{\partial \varphi}{\partial \nu}\left(w_{2}^{(2)}-w_{2}^{(1)}\right) d s_{x}
$$

holds for any $\varphi \in C^{2}(\bar{\Omega}), \varphi \geq 0$ on $\bar{\Omega}$. As before, for given $\eta \in C_{0}^{\infty}(\Omega) \eta \geq 0$ on $\Omega$, take $\varphi \in C^{2}(\bar{\Omega})$ as the solution of (2.3). Then we have

$$
\int_{\Omega}\left(w_{1}^{(1)}-w_{1}^{(2)}\right) \eta d x \geq 0
$$

and since $\eta \in C_{0}^{\infty}(\Omega), \eta \geq 0$ can be chosen arbitrary, we conclude that $w_{1}^{(1)} \geq w_{1}^{(2)}$ a.e. on $\Omega$. Similarly, for any $\zeta \in C^{\infty}(\partial \Omega), \zeta \geq 0$ on $\partial \Omega$, let $\varphi \in C^{2}(\bar{\Omega})$ be the solution to $(2.2)$. Then we have

$$
0 \geq \int_{\partial \Omega} \zeta\left(w_{2}^{(2)}-w_{2}^{(1)}\right) d s_{x}
$$

which implies that $w_{2}^{(2)} \leq w_{2}^{(1)}$ a.e. on $\partial \Omega$. By induction, we obtain

$$
\begin{array}{ll}
\bar{w}_{1}=w_{1}^{(1)} \geq w_{1}^{(2)} \geq \cdots \geq w_{1}^{(n)} \geq \cdots, & \text { a.e. on } \Omega, \\
\bar{w}_{2}=w_{2}^{(1)} \geq w_{2}^{(2)} \geq \cdots \geq w_{2}^{(n)} \geq \cdots, & \text { a.e. on } \partial \Omega .
\end{array}
$$

By Lemma 6 , we know $w_{1}^{(n)} \geq 0$ and $w_{2}^{(n)} \geq 0$. By the monotone convergence theorem, $w_{1}^{(n)}$ and $w_{2}^{(n)}$ converges to $u_{1}, u_{2}$ respectively in $L^{1}(\Omega)$ and $L^{1}(\partial \Omega)$. Since $f$ is increasing, we have also $f\left(w_{2}^{(n)}\right) \leq f\left(w_{2}^{(1)}\right) \in L^{1}(\partial \Omega)$ for any $n \in \mathbb{N}$, which leads to $f\left(u_{2}\right) \in L^{1}(\partial \Omega)$. Finally, it is easy to check that $u=\left(u_{1}, u_{2}\right)$ is a desired weak solution to $(1.1)_{\lambda}$.

Main result in this section is the following nonexistence result for $(1.1)_{\lambda}$ above the extremal parameter $\lambda^{*}$. See [3] Corollary 2, or [10] Theorem 3.8.

Theorem 9 Assume (1.2). If $\lambda>\lambda^{*}$, then there is no solution to $(1.1)_{\lambda}$, even in the weak sense in Definition 3.

Actually, we prove the following proposition. Theorem 9 is an easy consequence of this proposition and the definition of $\lambda^{*}(1.5)$. 
Proposition 10 Let $\lambda>0$ and assume that there exists a weak solution $u=\left(u_{1}, u_{2}\right) \in L^{1}(\Omega) \times L^{1}(\partial \Omega)$ to $(1.1)_{\lambda}$. Then for any $\alpha \in(0,1)$, the problem

$$
\begin{cases}-\Delta u+u=0 & \text { in } \Omega, \\ \frac{\partial u}{\partial \nu}=\alpha \lambda f(u) & \text { on } \partial \Omega,\end{cases}
$$

has a classical solution.

Proof. Let $u=\left(u_{1}, u_{2}\right) \in L^{1}(\Omega) \times L^{1}(\partial \Omega), u_{1}, u_{2} \geq 0$ be a weak solution to $(1.1)_{\lambda}$. Given $\alpha \in(0,1)$, define

$$
H(t)=\int_{0}^{t} \frac{d s}{\lambda f(s)}
$$

and

$$
\Phi(t)=H^{-1}(\alpha H(t))
$$

for $t \geq 0$. Then by an easy observation, we see

(i) $0=\Phi(0) \leq \Phi(t) \leq t$ for all $t \geq 0$,

(ii) $\Phi$ is increasing, concave, $\Phi^{\prime}(t) \leq 1$ for all $t \geq 0$,

(iii) if $\lim _{t \rightarrow+\infty} H(t)$ is finite, then $\lim _{t \rightarrow+\infty} \Phi(t)$ is also finite,

see [3]:Lemma 4. Also simple calculation shows

$$
\lambda \Phi^{\prime}(t) f(t)=\alpha \lambda f(\Phi(t))
$$

holds. Thus, by Lemma 7 and the relation (2.9), we see that $v=\left(v_{1}, v_{2}\right)=$ $\left(\Phi\left(u_{1}\right), \Phi\left(u_{2}\right)\right) \in L^{1}(\Omega) \times L^{1}(\partial \Omega)$ satisfies

$$
\int_{\Omega}(-\Delta \varphi+\varphi) v_{1} d x \geq \int_{\partial \Omega}\left\{\alpha \lambda f\left(v_{2}\right) \varphi-\frac{\partial \psi}{\partial \nu} v_{2}\right\} d s_{x}
$$

for any $\varphi \in C^{2}(\bar{\Omega}), \varphi \geq 0$ on $\bar{\Omega}$. That is, $v=\left(v_{1}, v_{2}\right)$ is a weak supersolution to $(1.1)_{\alpha \lambda}$.

Suppose first that

$$
\int_{0}^{\infty} \frac{d s}{f(s)}<+\infty
$$


In this case, by (iii) above, we have $\Phi(\infty)<\infty$, which implies $\left(v_{1}, v_{2}\right)=$ $\left(\Phi\left(u_{1}\right), \Phi\left(u_{2}\right)\right)$ be a bounded weak supersolution to $(1.1)_{\alpha \lambda}$. By Lemma 8, we have a weak solution to $(1.1)_{\alpha \lambda}$, which is bounded, hence classical solution. This proves Proposition in this case.

Next, consider the case

$$
\int_{0}^{\infty} \frac{d s}{f(s)}=+\infty
$$

In this case, we set $v^{(1)}=\left(v_{1}^{(1)}, v_{2}^{(1)}\right)=\left(\Phi\left(u_{1}\right), \Phi\left(u_{2}\right)\right) \in L^{1}(\Omega) \times L^{1}(\partial \Omega)$. Then by (i), we have $0 \leq v_{i}^{(1)} \leq u_{i}$ for $i=1,2$, and since $H$ is concave,

$$
H\left(u_{i}\right)-H\left(v_{i}^{(1)}\right) \leq H^{\prime}\left(v_{i}^{(1)}\right)\left(u_{i}-v_{i}^{(1)}\right)
$$

holds. By the definitions (2.7) and (2.8), we have

$$
H\left(v_{2}^{(1)}\right)=\alpha H\left(u_{2}\right), \quad \text { and } \quad H^{\prime}\left(v_{2}^{(1)}\right)=\frac{1}{\lambda f\left(v_{2}^{(1)}\right)} .
$$

Thus, we obtain

$$
\lambda(1-\alpha) H\left(u_{2}\right) \leq \frac{u_{2}-v_{2}^{(1)}}{f\left(v_{2}^{(1)}\right)},
$$

hence by the assumption $H(\infty)=+\infty$,

$$
\lambda(1-\alpha) f\left(v_{2}^{(1)}\right) \leq \frac{u_{2}}{H\left(u_{2}\right)} \leq C\left(1+u_{2}\right) \in L^{1}(\partial \Omega),
$$

which leads to $f\left(v_{2}^{(1)}\right) \in L^{1}(\partial \Omega)$. By Lemma $7, v^{(1)}=\left(v_{1}^{(1)}, v_{2}^{(1)}\right) \in L^{1}(\Omega) \times$ $L^{1}(\partial \Omega)$ is a weak supersolution of $(1.1)_{\alpha \lambda}$. Therefore by Lemma 8 , we obtain a weak solution $u^{(1)}=\left(u_{1}^{(1)}, u_{2}^{(1)}\right) \in L^{1}(\Omega) \times L^{1}(\partial \Omega)$ with the property $u_{i}^{(1)} \leq$ $v_{i}^{(1)}$ for $i=1,2$. Also since $f$ is positive and increasing, $0 \leq f\left(u_{2}^{(1)}\right) \leq$ $f\left(v_{2}^{(1)}\right) \in L^{1}(\partial \Omega)$. Hence by the elliptic $L^{1}$ estimate of Brezis and Strauss [5], we have $u_{1}^{(1)} \in W^{1, q}(\Omega)$ for any $1 \leq q<\frac{N}{N-1}$ and $u_{2}^{(1)} \in L^{p}(\partial \Omega)$ for any $1 \leq p<\frac{N-1}{N-2}$ (for any $p<\infty$ if $N=2$ ). Now, set $v^{(2)}=\left(v_{1}^{(2)}, v_{2}^{(2)}\right)=$ $\left(\Phi\left(u_{1}^{(1)}\right), \Phi\left(u_{2}^{(1)}\right)\right)$ and repeat the procedure. We confirm that $v^{(2)}$ is a weak supersolution to $(1.1)_{\alpha^{2} \lambda}$, and there exists a weak solution $u^{(2)}=\left(u_{1}^{(2)}, u_{2}^{(2)}\right)$ to $(1.1)_{\alpha^{2} \lambda}$ with the property that $0 \leq f\left(u_{2}^{(2)}\right) \leq f\left(v_{2}^{(2)}\right)$ a.e. on $\partial \Omega$,

$$
\lambda\left(1-\alpha^{2}\right) f\left(v_{2}^{(2)}\right) \leq \frac{u_{2}^{(1)}}{H\left(u_{2}^{(1)}\right)} \leq C\left(1+u_{2}^{(1)}\right) \in L^{p}(\partial \Omega),
$$


in particular, $f\left(u_{2}^{(2)}\right) \in L^{p}(\partial \Omega)$ for any $1 \leq p<\frac{N-1}{N-2}$ (for any $p<\infty$ if $N=2)$. Then elliptic $L^{p}$ estimates $([1,2])$ that $u_{1}^{(2)} \in W^{1, q}(\Omega)$ for any $q<\frac{N}{N-2}$ and the trace Sobolev embedding implies $u_{2}^{(2)} \in L^{p}(\partial \Omega)$ for any $p<\frac{N-1}{N-3}$ (for any $p<\infty$ if $N=3$ ). By iteration, we find a weak solution $u^{(k)}=\left(u_{1}^{(k)}, u_{2}^{(k)}\right)$ to the problem

$$
\begin{cases}-\Delta u^{(k)}+u^{(k)}=0 & \text { in } \Omega, \\ \frac{\partial u^{(k)}}{\partial \nu}=\alpha^{k} \lambda f\left(u^{(k)}\right) & \text { on } \partial \Omega,\end{cases}
$$

with the property that

$$
u_{1}^{(k)} \in W^{1, q}(\Omega), \quad \forall q<\frac{N}{N-k}, \quad u_{2}^{(k)} \in L^{p}(\partial \Omega), \quad \forall p<\frac{N-1}{N-(k+1)} .
$$

Thus after iterating $N$ times, we obtain that $u_{1}^{(k)} \in L^{\infty}(\Omega)$ and $u_{2}^{(k)} \in$ $L^{\infty}(\partial \Omega)$. That is, $u^{(k)}$ is a bounded, hence classical solution to $(1.1)_{\alpha^{k} \lambda}$. Since $\alpha \in(0,1)$ is arbitrary, we complete the proof.

\section{Regularity of extremal solutions}

In this section, we prove the extremal solution $u^{*}$ to our problem (1.1) is bounded for $N=2$. We follow the argument by Nedev [15], in which the extremal solution of (1.4) is bounded (hence regular by usual elliptic estimates) when $N \leq 3$. Recently, this result for the extremal solution of (1.4) is improved to $N=4$ by Villegas [18], which uses a key estimate by X. Cábre $[8]$.

Theorem 11 Let $u^{*}$ be the extremal solution to $(1.1)_{\lambda^{*}}$. Assume $f \in C^{2}([0,+\infty))$ satisfies (1.2), (1.3). Then we have:

(i) If $N=2$, then $u^{*} \in L^{\infty}(\Omega)$.

(ii) If $N \geq 3$, then $u^{*} \in L^{p}(\partial \Omega)$ for $1 \leq p<\frac{N-1}{N-3}$ (for any $1 \leq p<\infty$ when $N=3$ ) and $f\left(u^{*}\right) \in L^{p}(\partial \Omega)$ for any $1 \leq p<\frac{N-1}{N-2}$ (for any $1 \leq p<\infty$ when $N=2$ ).

(iii) $u^{*} \in W^{1, \gamma}(\Omega)$ for any $1 \leq \gamma<\frac{N}{N-2}$ when $N \geq 3$ (for any $1 \leq \gamma<\infty$ when $N=2)$. In particular, $u^{*} \in H^{1}(\Omega)$ if $N \leq 3$. 
Proof. We obtain several estimates of minimal solutions $u_{\lambda}$ to $(1.1)_{\lambda}$ which are independent of $\lambda \in\left(0, \lambda^{*}\right)$. Following Nedev [15], see also [10], we put

$$
g(t)=\int_{0}^{t}\left\{f^{\prime}(s)\right\}^{2} d s, \quad t \geq 0 .
$$

Since $f$ is $C^{2}, g$ is also a $C^{2}$ function. Multiplying $g\left(u_{\lambda}\right) \in C^{2}(\bar{\Omega})$ to the equation of $(1.1)_{\lambda}$ satisfied by $u_{\lambda}$ and integrating, we obtain

$$
\int_{\Omega} f^{\prime}\left(u_{\lambda}\right)^{2}\left|\nabla u_{\lambda}\right|^{2} d x=\lambda \int_{\partial \Omega} f\left(u_{\lambda}\right) g\left(u_{\lambda}\right) d s_{x}-\int_{\Omega} u_{\lambda} g\left(u_{\lambda}\right) d x .
$$

Recall the stability of $u_{\lambda}$ :

$$
\int_{\Omega}\left(|\nabla \varphi|^{2}+\varphi^{2}\right) d x \geq \lambda \int_{\partial \Omega} f^{\prime}\left(u_{\lambda}\right) \varphi^{2} d s_{x}
$$

holds for every $\varphi \in C^{1}(\bar{\Omega})$. Applying this inequality to $\varphi=\tilde{f}\left(u_{\lambda}\right), \tilde{f}(t)=$ $f(t)-f(0)$, we obtain

$$
\lambda \int_{\partial \Omega} f^{\prime}\left(u_{\lambda}\right) \tilde{f}\left(u_{\lambda}\right)^{2} d s_{x} \leq \int_{\Omega}\left(f^{\prime}\left(u_{\lambda}\right)^{2}\left|\nabla u_{\lambda}\right|^{2}+\tilde{f}\left(u_{\lambda}\right)^{2}\right) d x .
$$

By (3.1) and (3.2), we have

$$
\begin{aligned}
\lambda \int_{\partial \Omega}\left\{f^{\prime}\left(u_{\lambda}\right) \tilde{f}\left(u_{\lambda}\right)^{2}-\tilde{f}\left(u_{\lambda}\right) g\left(u_{\lambda}\right)\right\} d s_{x} & \leq \lambda f(0) \int_{\partial \Omega} g\left(u_{\lambda}\right) d s_{x} \\
& +\int_{\Omega}\left(\tilde{f}\left(u_{\lambda}\right)^{2}-u_{\lambda} g\left(u_{\lambda}\right)\right) d x .
\end{aligned}
$$

Let

$$
h(t)=\int_{0}^{t} f^{\prime}(s)\left(f^{\prime}(t)-f^{\prime}(s)\right) d s .
$$

Then we see $h(t) \geq 0$ and $f^{\prime}(t) \tilde{f}(t)^{2}-\tilde{f}(t) g(t)=\tilde{f}(t) h(t)$. Also if we put

$$
A(t)=\tilde{f}(t)^{2}-t g(t),
$$

then we see $A \in C^{2}([0, \infty)), A(0)=0, A^{\prime}(0)=0$ and

$$
A^{\prime \prime}(t)=2 f^{\prime \prime}(t)\left\{\tilde{f}(t)-t f^{\prime}(t)\right\} \leq 0,
$$


since by the convexity of $f$ and the assumption $f \in C^{2}$, we have $f^{\prime \prime}(t) \geq 0$ and $f^{\prime}(t) \geq \frac{f(t)-f(0)}{t}$ for $t>0$. Thus we obtain $A(t) \leq 0$ for all $t \geq 0$, which leads to

$$
\lambda \int_{\partial \Omega} \tilde{f}\left(u_{\lambda}\right) h\left(u_{\lambda}\right) d s_{x} \leq \lambda f(0) \int_{\partial \Omega} g\left(u_{\lambda}\right) d s_{x}
$$

from (3.3). By the same argument in [15], we have

$$
\lim _{t \rightarrow \infty} \frac{h(t)}{f^{\prime}(t)}=+\infty
$$

and

$$
g(t)=\int_{0}^{t}\left\{f^{\prime}(s)\right\}^{2} d s \leq \int_{0}^{t} f^{\prime}(s) f^{\prime}(t) d s \leq f^{\prime}(t) \tilde{f}(t),
$$

which with (3.5) implies

$$
\lim _{t \rightarrow \infty} \frac{\tilde{f}(t) h(t)}{g(t)}=+\infty
$$

From (3.4),(3.5) and (3.6), we have, as in [15],

$$
\int_{\partial \Omega} g\left(u_{\lambda}\right) d s_{x} \leq C, \quad \int_{\partial \Omega} \tilde{f}\left(u_{\lambda}\right) h\left(u_{\lambda}\right) d s_{x} \leq C
$$

and also

$$
\int_{\partial \Omega} \tilde{f}\left(u_{\lambda}\right) f^{\prime}\left(u_{\lambda}\right) d s_{x} \leq C, \quad \int_{\partial \Omega} \frac{\tilde{f}\left(u_{\lambda}\right)^{2}}{u_{\lambda}} d s_{x} \leq C
$$

for $C>0$ independent of $\lambda$. We prove here (3.7) only. Indeed, by (3.6), there exists $T>0$ such that $h(t) \tilde{f}(t) \geq 2 f(0) g(t)$ for all $t>T$. Let

$$
\partial \Omega_{\lambda, T}=\left\{x \in \partial \Omega \mid u_{\lambda}(x)>T\right\} .
$$

Then we have

$$
2 f(0) \int_{\partial \Omega_{\lambda, T}} g\left(u_{\lambda}\right) d s_{x} \leq \int_{\partial \Omega_{\lambda, T}} \tilde{f}\left(u_{\lambda}\right) h\left(u_{\lambda}\right) d s_{x} \leq f(0) \int_{\partial \Omega} g\left(u_{\lambda}\right) d s_{x}
$$

by (3.4). This implies

$$
\int_{\partial \Omega_{\lambda, T}} g\left(u_{\lambda}\right) d s_{x} \leq \int_{\partial \Omega \backslash \partial \Omega_{\lambda, T}} g\left(u_{\lambda}\right) d s_{x} \leq g(T)|\partial \Omega|
$$


and

$$
\int_{\partial \Omega} g\left(u_{\lambda}\right) d s_{x} \leq 2 g(T)|\partial \Omega|
$$

Backing to (3.4), we have (3.7).

From (3.8) and the assumption $\lim _{t \rightarrow \infty} \frac{\tilde{f}(t)}{t}=+\infty$, we obtain that

$$
\int_{\partial \Omega} f\left(u_{\lambda}\right) d s_{x} \leq C
$$

for some $C>0$ independent of $\lambda$. By the elliptic $L^{1}$ estimate of Brezis and Strauss [5], we have

$$
\begin{aligned}
& u_{\lambda} \in W^{1, q}(\Omega) \quad \text { for any } 1 \leq q<\frac{N}{N-1}, \quad \text { and } \\
& u_{\lambda} \in L^{p}(\partial \Omega) \quad \text { for any } 1 \leq p<\frac{N-1}{N-2}, \quad(1 \leq p<\infty \text { if } N=2) .
\end{aligned}
$$

Let $\alpha \in(0,1)$ and define

$$
\begin{aligned}
& A=\left\{x \in \partial \Omega \mid \tilde{f}\left(u_{\lambda}(x)\right) \leq u_{\lambda}(x)^{1 / \alpha}\right\}, \\
& B=\left\{x \in \partial \Omega \mid \tilde{f}\left(u_{\lambda}(x)\right)^{2} / u_{\lambda}(x)>\tilde{f}\left(u_{\lambda}(x)\right)^{2-\alpha}\right\} .
\end{aligned}
$$

Then by (3.8), we have

$$
\int_{B} \tilde{f}\left(u_{\lambda}(x)\right)^{2-\alpha} d s_{x} \leq C
$$

and

$$
\int_{A} \tilde{f}\left(u_{\lambda}\right)^{p} d s_{x} \leq \int_{A} u_{\lambda}^{p / \alpha} d s_{x} \leq C
$$

if $p / \alpha<\frac{N-1}{N-2}$. Choosing $\alpha \in(0,1)$ such that $2-\alpha=\frac{\alpha(N-1)}{N-2}$, i.e., $\alpha=\frac{2(N-2)}{2 N-3}$ $(\alpha>0$ is any small if $N=2)$, we see that

$$
\int_{\partial \Omega} \tilde{f}\left(u_{\lambda}\right)^{p} d s_{x} \leq C \quad \text { for } 1 \leq p<\frac{\alpha(N-1)}{N-2}=\frac{2 N-1}{2 N-3} .
$$

Then, elliptic $L^{p}$ estimate ([1], [2]) implies

$$
u_{\lambda} \in W^{1, \gamma}(\Omega) \quad \text { for } \gamma=\frac{N p}{N-1}, 1 \leq p<\frac{2(N-1)}{2 N-3},
$$


and by the trace Sobolev embedding $W^{1, \gamma}(\Omega) \hookrightarrow L^{\frac{(N-1) \gamma}{N-\gamma}}(\partial \Omega)$,

$$
u_{\lambda} \in L^{p}(\partial \Omega) \quad \text { for any } 1 \leq p<\frac{2(N-1)}{2 N-5},(1 \leq p \leq \infty \text { if } N=2) .
$$

Now, we use a bootstrap argument. Assume we obtain that $u_{\lambda} \in L^{p}(\partial \Omega)$ for $p<p_{0}$. We choose $\alpha \in(0,1)$ as $2-\alpha=\alpha p_{0}$, i.e., $\alpha=\frac{2}{1+p_{0}}$. Then elliptic $L^{p}$ estimate and trace Sobolev embedding imply that $u_{\lambda} \in W^{1, \gamma}(\Omega) \hookrightarrow$ $L^{\frac{(N-1) \gamma}{N-\gamma}}(\partial \Omega)$, where $\gamma=\frac{N p}{N-1}$ so $\frac{(N-1) \gamma}{N-\gamma}=\frac{(N-1) p}{N-1-p}$. Also $\left\|f\left(u_{\lambda}\right)\right\|_{L^{p}(\partial \Omega)} \leq C$ for any $p<\alpha p_{0}$. Note that when $p<\frac{2 p_{0}}{1+p_{0}}$, then $\frac{(N-1) p}{N-1-p}<\frac{2(N-1) p_{0}}{N-1+(N-3) p_{0}}$. Let us define

$$
p_{\infty}=\frac{2(N-1) p_{\infty}}{N-1+(N-3) p_{\infty}},
$$

that is, $p_{\infty}=\frac{N-1}{N-3}$. Then we obtain $\left\|u_{\lambda}\right\|_{L^{p}(\partial \Omega)} \leq C$ independent of $\lambda$ for any $p<\frac{N-1}{N-3}$ and also $\left\|f\left(u_{\lambda}\right)\right\|_{L^{p}(\partial \Omega)} \leq C$ for any $p<\frac{2 p_{\infty}}{1+p_{\infty}}=\frac{N-1}{N-2}$. Thus by elliptic estimates, we have $u_{\lambda} \in W^{1, \gamma}(\Omega)$ for $\gamma=\frac{N p}{N-1}, p<\frac{N-1}{N-2}$. Thus $u_{\lambda} \in W^{1, \gamma}(\Omega)$ for any $\gamma<\frac{N}{N-2}$ when $N \geq 3$.

For typical nonlinearities such as $f(u)=e^{u}$ or $f(u)=(1+u)^{p}$ for $p>1$, we improve the above result as follows:

Proposition 12 Let $u^{*}$ be the extremal solution to $(1.1)_{\lambda^{*}}$ with $f(u)=e^{u}$. Then if $N \leq 5$, we have $u^{*} \in L^{\infty}(\Omega)$.

Proposition 13 Let $u^{*}$ be the extremal solution to $(1.1)_{\lambda^{*}}$ with $f(u)=(1+$ $u)^{p}$ for $p>1$. Define

$$
N_{p}=4+2\left(\frac{1}{p-1}+\sqrt{1+\frac{1}{p-1}}\right) .
$$

Then if $N<N_{p}$, we have $u^{*} \in L^{\infty}(\Omega)$.

In particular, if $N \leq 6$, or $N \geq 7$ and $1<p<p_{+}(N):=\frac{N^{2}-6 N+6+2 \sqrt{2 N-3}}{(N-2)(N-6)}$, then $u^{*} \in L^{\infty}(\Omega)$.

Note that for our problem (1.1), we do not know any information of the explicit singular extremal solutions even when $\Omega=B$ is a ball and $f$ is one of the above nonlinearities. 
Proof of Proposition 12. We follow the arguments in [9], [14] with some modifications for out context. Recall the minimal solution $u_{\lambda}$ satisfies the stability inequality

$$
\int_{\Omega}\left(|\nabla \varphi|^{2}+\varphi^{2}\right) d x \geq \lambda \int_{\partial \Omega} e^{u_{\lambda}} \varphi^{2} d s_{x}, \quad \forall \varphi \in C^{1}(\bar{\Omega})
$$

and the weak form of the equation

$$
\int_{\Omega}\left(\nabla \psi \cdot \nabla u_{\lambda}+u_{\lambda} \psi\right) d x=\lambda \int_{\partial \Omega} e^{u_{\lambda}} \psi d s_{x}, \quad \forall \psi \in C^{1}(\bar{\Omega}) .
$$

We put $\varphi=e^{t u_{\lambda}}$ and $\psi=e^{2 t u_{\lambda}}$, where $t>0$. Testing with them we have

$$
\int_{\Omega}\left(t^{2} e^{2 t u_{\lambda}}\left|\nabla u_{\lambda}\right|^{2}+e^{2 t u_{\lambda}}\right) d x \geq \lambda \int_{\partial \Omega} e^{(2 t+1) u_{\lambda}} d s_{x}
$$

and

$$
\int_{\Omega}\left(2 t e^{2 t u_{\lambda}}\left|\nabla u_{\lambda}\right|^{2}+u_{\lambda} e^{2 t u_{\lambda}}\right) d x=\lambda \int_{\partial \Omega} e^{(2 t+1) u_{\lambda}} d s_{x} .
$$

Combining these, we obtain

$$
2 \int_{\Omega} e^{2 t u_{\lambda}} d x-t \int_{\Omega} u_{\lambda} e^{2 t u_{\lambda}} d x \geq \lambda(2-t) \int_{\partial \Omega} e^{(2 t+1) u_{\lambda}} d s_{x} .
$$

Since $(0,+\infty) \ni s \mapsto(2-t s) e^{2 t s}$ is bounded from above for $t>0$, the left hand side is bounded when $\lambda \uparrow \lambda^{*}$. Thus for any $0<t<2$, we have $e^{u_{\lambda}}$ is uniformly bounded in $L^{2 t+1}(\partial \Omega)$, and the elliptic estimate implies that $\left\|u_{\lambda}\right\|_{W^{1, \frac{N}{N-1}(2 t+1)}(\Omega)} \leq C$ uniformly in $\lambda$. Sobolev embedding assures that $\left\|u_{\lambda}\right\|_{L^{\infty}(\Omega)} \leq C$ uniformly in $\lambda$ if $2 t+1>N-1$. Since $t$ can be chosen arbitrary near to 2 , this shows that $u^{*} \in L^{\infty}(\Omega)$ if $N<6$.

Proof of Proposition 13. Again, minimal solution $u_{\lambda}$ satisfies the stability inequality

$$
\int_{\Omega}\left(|\nabla \varphi|^{2}+\varphi^{2}\right) d x \geq \lambda \int_{\partial \Omega} p\left(1+u_{\lambda}\right)^{p-1} \varphi^{2} d s_{x}, \quad \forall \varphi \in C^{1}(\bar{\Omega})
$$

and the weak form of the equation

$$
\int_{\Omega}\left(\nabla \psi \cdot \nabla u_{\lambda}+u_{\lambda} \psi\right) d x=\lambda \int_{\partial \Omega}\left(1+u_{\lambda}\right)^{p} \psi d s_{x}, \quad \forall \psi \in C^{1}(\bar{\Omega}) .
$$


In this case, choosing $\varphi=\left(1+u_{\lambda}\right)^{t p+\frac{1}{2}}$ and $\psi=\left(1+u_{\lambda}\right)^{2 t p}$ for $t>0$, we have $\int_{\Omega}\left\{\left(t p+\frac{1}{2}\right)^{2}\left(1+u_{\lambda}\right)^{2 t p-1}\left|\nabla u_{\lambda}\right|^{2}+\left(1+u_{\lambda}\right)^{2 t p+1}\right\} d x \geq \lambda p \int_{\partial \Omega}\left(1+u_{\lambda}\right)^{p(2 t+1)} d s_{x}$ and

$$
\int_{\Omega}\left\{2 t p\left(1+u_{\lambda}\right)^{2 t p-1}\left|\nabla u_{\lambda}\right|^{2}+u_{\lambda}\left(1+u_{\lambda}\right)^{2 t p}\right\} d x=\lambda \int_{\partial \Omega}\left(1+u_{\lambda}\right)^{p(2 t+1)} d s_{x} .
$$

Combining these, we have

$$
\begin{aligned}
& \int_{\Omega}\left\{\left(t p+\frac{1}{2}\right)^{2}\left(1+u_{\lambda}\right)^{2 t p}-\left(t p-\frac{1}{2}\right)^{2}\left(1+u_{\lambda}\right)^{2 t p+1}\right\} d x \\
& \geq \lambda\left\{2 t p^{2}-\left(t p+\frac{1}{2}\right)^{2}\right\} \int_{\partial \Omega}\left(1+u_{\lambda}\right)^{p(2 t+1)} d s_{x} .
\end{aligned}
$$

Since $(0,+\infty) \ni s \mapsto A(1+s)^{2 t p}-B(1+s)^{2 t p+1}$ is bounded from above for $A, B>0$, the left hand side is bounded when $\lambda \uparrow \lambda^{*}$. Therefore, we have a uniform bound $\left\|\left(1+u_{\lambda}\right)^{p}\right\|_{L^{2 t+1}(\partial \Omega)} \leq C$ when $2 t p^{2}-\left(t p+\frac{1}{2}\right)^{2}>$ 0 . This quadratic inequality with respect to $t$ is equivalent to that $t \in$ $\left(\frac{2 p-1-2 \sqrt{p(p-1)}}{2 p}, \frac{2 p-1+2 \sqrt{p(p-1)}}{2 p}\right)$, that is,

$$
\frac{3 p-1-2 \sqrt{p(p-1)}}{p}<2 t+1<\frac{3 p-1+2 \sqrt{p(p-1)}}{p} \text {. }
$$

Now, we use a bootstrap argument. If $\left(1+u_{\lambda}\right)^{p} \in L^{q}(\partial \Omega)$, elliptic estimate and trace Sobolev embedding imply that $u_{\lambda} \in W^{1, \frac{N}{N-1} q}(\Omega) \hookrightarrow L^{\frac{(N-1) q}{N-1-q}}(\partial \Omega)$. Define $\left\{q_{k}\right\}_{k \in \mathbb{N}}$ as

$$
\left\{\begin{array}{l}
q_{1}=2 t+1 \\
\frac{1}{q_{k+1}}=p\left(\frac{1}{q_{k}}-\frac{1}{N-1}\right), \quad(k=1,2, \cdots) .
\end{array}\right.
$$

We easily obtain that $\frac{1}{q_{k}}=p^{k-1}\left(\frac{1}{q_{1}}-\frac{p}{(N-1)(p-1)}\right)+\frac{p}{(N-1)(p-1)}$, hence if

$$
\frac{1}{q_{1}}<\frac{p}{(N-1)(p-1)}
$$


then there exists some $k \in \mathbb{N}$ such that $\frac{1}{q_{k+1}}<0$, which implies $q_{k}>N-1$ and $u_{\lambda} \in W^{1, \frac{N}{N-1} q_{k}}(\Omega) \hookrightarrow L^{\infty}(\Omega)$, which ends the proof. Since $q_{1}=2 t+1$ can be chosen arbitrary close to the number $\frac{3 p-1+2 \sqrt{p(p-1)}}{p},(3.10)$ is satisfied when

$$
(N-1)\left(1-\frac{1}{p}\right)<\frac{3 p-1+2 \sqrt{p(p-1)}}{p},
$$

which is equivalent to $N<N_{p}$ where $N_{p}$ is defined in (3.9). Since $N_{p}$ is decreasing with respect to $p$ and $N_{p} \rightarrow 6$ as $p \rightarrow \infty$, we have $N_{p}>6$ for any $p>1$. Also we can check that the inequality $N<N_{p}$ is equivalent to $1<p<p_{+}(N)=\frac{N^{2}-6 N+6+2 \sqrt{2 N-3}}{(N-2)(N-6)}$ when $N \geq 7$. This proves Proposition 13.

\section{Uniqueness of weak extremal solutions}

In this section, following the argument of Martel [13], see also [10], we show the uniqueness of extremal solution even in the weak sense, as described below.

Theorem 14 Assume $f \in C^{2}([0,+\infty))$ satisfies (1.2), (1.3). Let $\lambda^{*}$ be defined in (1.5). Assume (1.1) $\lambda_{\lambda^{*}}$ has a weak supersolution $w=\left(w_{1}, w_{2}\right) \in$ $L^{1}(\Omega) \times L^{1}(\partial \Omega)$, in the sense that $f\left(w_{2}\right) \in L^{1}(\partial \Omega)$ and

$$
\int_{\Omega}(-\Delta \varphi+\varphi) w_{1} d x \geq \int_{\partial \Omega}\left\{\lambda^{*} f\left(w_{2}\right) \varphi-\frac{\partial \varphi}{\partial \nu} w_{2}\right\} d s_{x}
$$

for any $\varphi \in C^{2}(\bar{\Omega}), \varphi \geq 0$ on $\bar{\Omega}$. Then $\left(w_{1}, w_{2}\right)=\left(\left(u^{*}\right)_{1},\left(u^{*}\right)_{2}\right)$, where $u^{*}$ is defined by (1.10). As a consequence, the extremal solution $u^{*}$ is the unique weak solution to $(1.1)_{\lambda^{*}}$.

Proof. By assumption and Lemma 8, there exists a weak solution $u$ to $(1.1)_{\lambda^{*}}$. We argue by contradiction and assume that $u \not \equiv u^{*}, u>u^{*}$ in $\bar{\Omega}$. We divide the proof into several steps.

Step 1. There exists a strict supersolution $v$ to $(1.1)_{\lambda^{*}}$.

Indeed, the convexity of $f$ implies that $u_{t}=t u^{*}+(1-t) u$ is a supersolution of $(1.1)_{\lambda^{*}}$ for any $t \in(0,1)$. Suppose on the contrary that $u_{t}$ is a solution to 
$(1.1)_{\lambda^{*}}$ for all $t \in(0,1)$. This implies that there is a set $\mathcal{N} \subset \partial \Omega$ with $(N-1)$ dimensional measure 0 such that $f\left(u_{t}(x)\right)=t f\left(u^{*}(x)\right)+(1-t) f(u(x))$ for any $x \in \partial \Omega \backslash \mathcal{N}$ and for all $t \in(0,1)$. Thus $f$ is linear on the interval $\left[u^{*}(x), u(x)\right]$ for such $x$. By the same argument of [10] p.148, which uses the regularity of the extremal solution $u^{*}$ as described in Theorem 11, we obtain that $u^{*}(\partial \Omega)$ is dense in the interval $\left[\operatorname{essinf}_{\partial \Omega} u^{*}, \operatorname{ess}_{\sup } \operatorname{su}_{\partial \Omega} u^{*}\right]$ and $\cup_{x \in \partial \Omega \backslash \mathcal{N}}\left[u^{*}(x), u(x)\right]$ is also an interval. This implies $u^{*}$ is a solution to

$$
\begin{cases}-\Delta u+u=0 & \text { in } \Omega, \\ \frac{\partial u}{\partial \nu}=\lambda^{*}(a u+b) & \text { on } \partial \Omega\end{cases}
$$

for some $a, b \in \mathbb{R}$. Assumption $f(0)>0$ implies $b>0$. In this linear case, we easily see that $\lambda^{*}=\frac{\lambda_{1}}{a}$, where $\lambda_{1}$ is the first eigenvalue of the problem (1.11). Regularity theory assures that $u^{*}$ is a classical solution. Thus if we multiply the equation by $\varphi_{1}$ the first eigenfunction of (1.11) with the normalization $\int_{\partial \Omega} \varphi_{1} d s_{x}=1$, we have

$$
\int_{\partial \Omega} \lambda^{*}(a u+b) \varphi_{1} d s_{x}=\int_{\partial \Omega} \lambda_{1} u \varphi_{1} d s_{x}
$$

Thus we obtain $b=0$, a contradiction.

Step 2. There is an $\varepsilon>0$ such that

$$
\begin{cases}-\Delta u+u=0 & \text { in } \Omega, \\ \frac{\partial u}{\partial \nu}=\lambda^{*} f(u)+\varepsilon & \text { on } \partial \Omega\end{cases}
$$

has a weak supersolution $w$.

Indeed, by Step 1, we have a strict supersolution $v$ to $(1.1)_{\lambda^{*}}$. Let $V$ be the solution to the linear problem

$$
\begin{cases}-\Delta V+V=0 & \text { in } \Omega, \\ \frac{\partial V}{\partial \nu}=\lambda^{*} f(v) & \text { on } \partial \Omega,\end{cases}
$$

and $\psi$ is a solution of (2.4). Then the maximum principle implies $v-V \geq \varepsilon \psi$ on $\bar{\Omega}$ for sufficiently small $\varepsilon>0$. Define $w=V+\varepsilon \psi$. Then we see $w \leq v$ and

$$
\frac{\partial w}{\partial \nu}=\lambda^{*} f(v)+\varepsilon \geq \lambda^{*} f(w)+\varepsilon
$$


by the monotonicity of $f$. Thus $w$ is a weak supersolution.

Step 3. Let $\varepsilon_{1} \in(0, \varepsilon)$, where $\varepsilon>0$ is a constant in Step 2. Then there exists a bounded (classical) solution to

$$
\begin{cases}-\Delta u+u=0 & \text { in } \Omega, \\ \frac{\partial u}{\partial \nu}=\lambda^{*} f(u)+\varepsilon_{1} & \text { on } \partial \Omega .\end{cases}
$$

The proof of this fact is quite similar to that of Proposition 10. Indeed, let us define

$$
H_{\varepsilon}(t)=\int_{0}^{t} \frac{d s}{\lambda^{*} f(s)+\varepsilon}
$$

and

$$
\Phi(t)=H_{\varepsilon_{1}}^{-1}\left(H_{\varepsilon}(t)\right)
$$

for $t \geq 0$. Put $v=\Phi(w)$ where $w$ is a weak supersolution in Step 2. It is enough to consider the case when $\int_{0}^{\infty} \frac{d s}{f(s)}=+\infty$, because otherwise, we find as before that $v=\Phi(w)$ is a bounded weak supersolution to (4.1) and Lemma 8 yields the result. We see $v \leq w$ and since $H_{\varepsilon}$ is concave,

$$
\frac{H_{\varepsilon}(w)-H_{\varepsilon}(v)}{w-v} \leq H_{\varepsilon}^{\prime}(v)=\frac{1}{\lambda^{*} f(v)+\varepsilon}
$$

Also since $H_{\varepsilon}(w)=H_{\varepsilon_{1}}(v)$, we have

$$
\begin{aligned}
H_{\varepsilon}(w)-H_{\varepsilon}(v)=H_{\varepsilon_{1}}(v)-H_{\varepsilon}(v) & =\int_{0}^{v}\left(\frac{1}{\lambda^{*} f(s)+\varepsilon_{1}}-\frac{1}{\lambda^{*} f(s)+\varepsilon}\right) d s \\
& \geq\left(\varepsilon-\varepsilon_{1}\right) \int_{0}^{v} \frac{1}{\left(\lambda^{*} f(s)+1\right)^{2}} d s .
\end{aligned}
$$

From these, we obtain $\lambda^{*} f(v)+\varepsilon \leq \frac{C(1+w)}{\varepsilon-\varepsilon_{1}} \in L^{1}(\partial \Omega)$. This and the bootstrap argument as in Proposition 10 yield the proof of Step 3.

Let $u$ be the bounded solution obtained in Step 3 and let $\lambda^{\prime}>\lambda^{*}$. Define

$$
U=\frac{\lambda^{\prime}}{\lambda^{*}} u-\varepsilon_{1} \psi
$$

where $\psi$ is a solution to (2.4). Then we see $\frac{\partial U}{\partial \nu}=\lambda^{\prime} f(u)+\frac{\lambda^{\prime}}{\lambda^{*}} \varepsilon_{1}-\varepsilon_{1} \geq \lambda^{\prime} f(u)$ on $\partial \Omega$. Choose $\frac{\lambda^{\prime}}{\lambda^{*}}>1$ sufficiently close to 1 in order to assure $U \leq u$ on $\bar{\Omega}$ 
(note that $u$ is bounded), then we see $U$ is a bounded supersolution to (1.1) for $\lambda=\lambda^{\prime}$. By Lemma 8, we have a classical solution to $(1.1)_{\lambda^{\prime}}$ for $\lambda^{\prime}>\lambda^{*}$, contradicting to the definition of $\lambda^{*}$.

As an application of Theorem 14, we show a characterization of the unbounded extremal solutions in the energy class $H^{1}(\Omega)$.

Theorem 15 Let $u \in H^{1}(\Omega), u \notin L^{\infty}(\partial \Omega)$, be a singular weak solution to $(1.1)_{\lambda}$ where $f$ is as in Theorem 14. Then the followings are equivalent:

(i) $f^{\prime}(u) \in L^{1}(\Omega)$ and

$$
\int_{\Omega}\left(|\nabla \varphi|^{2}+\varphi^{2}\right) d x \geq \lambda \int_{\partial \Omega} f^{\prime}(u) \varphi^{2} d s_{x}
$$

holds for every $\varphi \in C^{1}(\bar{\Omega}), \varphi \not \equiv 0$.

(ii) $\lambda=\lambda^{*}$ and $u=u^{*}$.

Proof. The implication $(i i) \Longrightarrow(i)$ follows easily by the stability property of the minimal solutions $u_{\lambda}$ and Fatou's lemma.

Let us prove $(i) \Longrightarrow(i i)$. Since no solution exists for $\lambda>\lambda^{*}$ by Theorem 9, we have $\lambda \leq \lambda^{*}$. Assume the contrary that $\lambda<\lambda^{*}$. By the density argument, we can take the test function $\varphi=u-u_{\lambda} \in H^{1}(\Omega)$. Note that here we have used the assumption $u \in H^{1}(\Omega)$. Also the assumption $u \notin L^{\infty}(\partial \Omega)$ implies that $u-u_{\lambda} \not \equiv 0$. Combining the equation satisfied by $u-u_{\lambda}$ with (i), we get

$$
\begin{aligned}
\lambda \int_{\partial \Omega}\left(f(u)-f\left(u_{\lambda}\right)\right)\left(u-u_{\lambda}\right) d s_{x} & =\int_{\Omega}\left(\left|\nabla\left(u-u_{\lambda}\right)\right|^{2}+\left(u-u_{\lambda}\right)^{2}\right) d x \\
& \geq \lambda \int_{\partial \Omega} f^{\prime}(u)\left(u-u_{\lambda}\right)^{2} d s_{x}
\end{aligned}
$$

which implies

$$
\lambda \int_{\partial \Omega}\left(u-u_{\lambda}\right)\left(f(u)-f\left(u_{\lambda}\right)-f^{\prime}(u)\left(u-u_{\lambda}\right)\right) d s_{x} \geq 0 .
$$

Since the integrand is non positive by the convexity of $f$, we conclude that $f(u)=f\left(u_{\lambda}\right)+f^{\prime}(u)\left(u-u_{\lambda}\right)$ a.e. on $\partial \Omega$. This implies that $f$ is linear on 
intervals of the form $\left[u_{\lambda}(x), u(x)\right]$ for a.e. $x \in \partial \Omega$. Now, since $u$ is unbounded on $\partial \Omega$, the union of these intervals is an interval of the form $[A,+\infty]$ and $f$ is linear on this interval. But this contradicts to the superlinearity at $\infty$ of $f$ in (1.3). Thus we have $\lambda=\lambda^{*}$. Finally, by the uniqueness of extremal solution $u^{*}$ in Theorem 14, we conclude $u=u^{*}$.

\section{Weak eigenfunctions for the extremal lin- earized problem}

In this section, we prove the following theorem, which is a natural extension of the result by Cabré and Martel [7] to our case.

Theorem 16 Let $f$ be as in Theorem 14. Then there exists a function $\varphi \geq 0$, $\varphi \not \equiv 0$, such that $\varphi \in W^{1, q}(\Omega)$ for any $1 \leq q<\frac{N}{N-1}, f^{\prime}\left(u^{*}\right) \varphi \in L^{1}(\partial \Omega)$ and

$$
\int_{\Omega}(-\Delta \zeta+\zeta) \varphi d x=\int_{\partial \Omega}\left\{\lambda^{*} f^{\prime}\left(u^{*}\right) \varphi \zeta-\frac{\partial \zeta}{\partial \nu} \varphi\right\} d s_{x}
$$

for all $\zeta \in C^{2}(\bar{\Omega})$. That is, there exists a weak solution to the linearized problem around the extremal solution $u^{*}$ :

$$
\begin{cases}-\Delta \varphi+\varphi=0 & \text { in } \Omega, \\ \frac{\partial \varphi}{\partial \nu}=\lambda^{*} f^{\prime}\left(u^{*}\right) \varphi & \text { on } \partial \Omega .\end{cases}
$$

First, we need a lemma.

Lemma 17 Let $\left\{u_{n}\right\} \subset C^{2}(\bar{\Omega})$ be a sequence of functions such that

$$
\begin{cases}-\Delta u_{n}+u_{n}=0 & \text { in } \Omega, \\ \frac{\partial u_{n}}{\partial \nu} \geq 0 & \text { on } \partial \Omega .\end{cases}
$$

Assume $\left\|u_{n}\right\|_{L^{1}(\partial \Omega)} \leq C$ for some $C>0$ independent of $n$. Then there exists a subsequence (denoted again by $u_{n}$ ) and $u \in W^{1, q}(\Omega)$ such that

$$
\begin{aligned}
& u_{n} \rightarrow u \quad \text { weakly in } W^{1, q}(\Omega), 1<q<\frac{N}{N-1}, \\
& u_{n} \rightarrow u \quad \text { strongly in } L^{p}(\partial \Omega), 1 \leq p<\frac{N-1}{N-2} .
\end{aligned}
$$


Moreover, for any $1 \leq p<\frac{N-1}{N-2}$, there exists a constant $C_{p}>0$ depending only on $p$ such that

$$
\left\|u_{n}\right\|_{L^{p}(\partial \Omega)} \leq C_{p}\left\|u_{n}\right\|_{L^{1}(\partial \Omega)}
$$

holds true for any $n \in \mathbb{N}$.

Proof. First we prove the a priori estimate (5.1) by a duality argument. For $\eta \in C^{\infty}(\partial \Omega)$ be given, let $\zeta \in C^{2}(\bar{\Omega})$ be a solution to

$$
\begin{cases}-\Delta \zeta+\zeta=0 & \text { in } \Omega \\ \frac{\partial \zeta}{\partial \nu}=\eta & \text { on } \partial \Omega\end{cases}
$$

Let $p \in\left(1, \frac{N-1}{N-2}\right)$. Then the Hölder conjugate exponent $p^{\prime}=\frac{p}{p-1}>N-1$. Elliptic estimate $([1,2])$ implies that $\|\zeta\|_{W^{1, \gamma}(\Omega)} \leq C\|\eta\|_{L^{p^{\prime}}(\partial \Omega)}$ where $\gamma=$ $\frac{N p^{\prime}}{N-1}>N$. Since $W^{1, \gamma}(\Omega) \hookrightarrow C^{\alpha}(\bar{\Omega})$ for $\alpha=1-\frac{N}{\gamma} \in(0,1)$, we have $|\zeta|(x) \leq C\|\eta\|_{L^{p^{\prime}(\partial \Omega)}} \varphi_{1}(x), x \in \bar{\Omega}$, where $\varphi_{1}$ denotes the first eigenfunction of the problem (1.11). By Green's identity, we have

$$
\begin{aligned}
& \left|\int_{\partial \Omega} u_{n} \eta d s_{x}\right|=\left|\int_{\partial \Omega} u_{n} \frac{\partial \zeta}{\partial \nu} d s_{x}\right| \\
& =\left|\int_{\partial \Omega}\left(\frac{\partial u_{n}}{\partial \nu}\right) \zeta d s_{x}-\int_{\Omega}(\Delta \zeta-\zeta) u_{n}-\left(\Delta u_{n}-u_{n}\right) \zeta d x\right| \\
& \leq \int_{\partial \Omega}\left(\frac{\partial u_{n}}{\partial \nu}\right)|\zeta| d s_{x} \leq C\|\eta\|_{L^{p^{\prime}}(\partial \Omega)} \int_{\partial \Omega}\left(\frac{\partial u_{n}}{\partial \nu}\right) \varphi_{1} d s_{x} \\
& =C\|\eta\|_{L^{p^{\prime}}(\partial \Omega)} \lambda_{1} \int_{\partial \Omega} u_{n} \varphi_{1} d s_{x} \leq C\|\eta\|_{L^{p^{\prime}}(\partial \Omega)} \lambda_{1}\left\|\varphi_{1}\right\|_{L^{\infty}(\partial \Omega)}\left\|u_{n}\right\|_{L^{1}(\partial \Omega)} .
\end{aligned}
$$

Since $\eta \in C^{\infty}(\partial \Omega)$ is arbitrary, we obtain (5.1) by duality.

Now, let $\psi>0$ be the solution of (2.4). Then we have

$$
\int_{\partial \Omega}\left(\frac{\partial u_{n}}{\partial \nu}\right) \psi d s_{x}=\int_{\partial \Omega}\left(\frac{\partial \psi}{\partial \nu}\right) u_{n} d s_{x}=\int_{\partial \Omega} u_{n} d s_{x}
$$

hence $\left\|\frac{\partial u_{n}}{\partial \nu}\right\|_{L^{1}(\partial \Omega)} \leq \frac{\left\|u_{n}\right\|_{L^{1}(\partial \Omega)}}{\min _{\partial \Omega} \psi} \leq C$, where $C$ is independent of $n$ by the assumption. Thus, by Brezis-Strauss estimate [5], we confirm that $\left\|u_{n}\right\|_{W^{1, q}(\Omega)} \leq$ $C\left\|\frac{\partial u_{n}}{\partial \nu}\right\|_{L^{1}(\partial \Omega)} \leq C$ for any $1 \leq q<\frac{N}{N-1}$ and there exists a subsequence such that $u_{n} \rightarrow u$ in $W^{1, q}(\Omega)$ for $1<q<\frac{N}{N-1}$ for some $u \in W^{1, q}(\Omega)$. Since the 
trace Sobolev embedding $W^{1, q}(\Omega) \hookrightarrow L^{p}(\partial \Omega)$ is compact if $1 \leq p<\frac{(N-1) q}{N-q}$, we conclude that $\left\|u_{n}-u\right\|_{L^{p}(\partial \Omega)} \rightarrow 0$ for $1 \leq p<\frac{N-1}{N-2}$.

Now, we prove Theorem 16 .

Proof. As in [7], we divide the proof into several steps.

Step 1. For $n \in \mathbb{N}$, define a sequence of functions of asymptotically linear approximations of $f$ as

$$
f_{n}(s)= \begin{cases}f(s) & \text { if } s \leq n, \\ f(n)+f^{\prime}(n)(s-n) & \text { if } s>n\end{cases}
$$

and consider the approximated problem

$$
\begin{cases}-\Delta u+u=0 & \text { in } \Omega \\ \frac{\partial u}{\partial \nu}=\lambda f_{n}(u) & \text { on } \partial \Omega .\end{cases}
$$

Denote

$$
\lambda_{n}^{*}=\sup \left\{\lambda>0:(5.2)_{\lambda} \text { admits a minimal classical solution } \in C^{2}(\bar{\Omega})\right\},
$$

and let $u_{n, \lambda}$ be a classical minimal solution to (5.2) for $\lambda<\lambda_{n}^{*}$. Note that $f_{n}(0)>0$, increasing and convex, the above extremal parameter $\lambda_{n}^{*}$ is finite and the existence of minimal solution is assured by the standard method. Note also that $f_{n} \leq f_{n+1} \leq f$, hence $\lambda^{*} \leq \lambda_{n+1}^{*} \leq \lambda_{n}^{*}$ for any $n \in \mathbb{N}$. Though $f_{n}$ does not satisfy the superlinear condition at $\infty$, we claim that the pointwise limit

$$
u_{n}^{*}(x)=\lim _{\lambda \uparrow \lambda_{n}^{*}} u_{n, \lambda}(x)
$$

is a classical solution of (5.2) $)_{\lambda_{n}^{*}}$ for $n$ large. Indeed, take $\lambda \in\left(\lambda^{*} / 2, \lambda^{*}\right)$ and let $u_{n, \lambda}$ be the minimal solution to $(5.2)_{\lambda}$. Multiplying the equation satisfied by $u_{n, \lambda}$ by $\varphi_{1}$, where $\varphi_{1}$ is the first eigenfunction of (1.11), which is normalized as $\int_{\partial \Omega} \varphi_{1} d s_{x}=1$, we obtain that

$$
\begin{aligned}
& \lambda_{1} \int_{\partial \Omega} \varphi_{1} u_{n, \lambda} d s_{x}=\lambda \int_{\partial \Omega} f_{n}\left(u_{n, \lambda}\right) \varphi_{1} d s_{x} \\
& \geq \lambda f_{n}\left(\int_{\partial \Omega} \varphi_{1} u_{n, \lambda} d s_{x}\right)>\frac{\lambda^{*}}{2} f_{n}\left(\int_{\partial \Omega} \varphi_{1} u_{n, \lambda} d s_{x}\right) .
\end{aligned}
$$


Here we have used Jensen's inequality for convex functions $f_{n}$. Thus we have

$$
a_{n, \lambda} \geq\left(\frac{\lambda^{*}}{2 \lambda_{1}}\right) f_{n}\left(a_{n, \lambda}\right)
$$

where we put $a_{n, \lambda}=\int_{\partial \Omega} \varphi_{1} u_{n, \lambda} d s_{x}$. On the other hand, (1.3) implies that $f(n)>\left(\frac{2 \lambda_{1}}{\lambda^{*}}\right) n$ and $f^{\prime}(n)>\left(\frac{2 \lambda_{1}}{\lambda^{*}}\right)$ for $n$ sufficiently large. Assume the contrary that $f_{n}\left(a_{n, \lambda}\right)=f^{\prime}(n)\left(a_{n, \lambda}-n\right)+f(n)$ for some $n \in \mathbb{N}$ sufficiently large. Then we have, since $a_{n, \lambda}>n$,

$$
\begin{aligned}
& a_{n, \lambda} \geq\left(\frac{\lambda^{*}}{2 \lambda_{1}}\right) f_{n}\left(a_{n, \lambda}\right)=\left(\frac{\lambda^{*}}{2 \lambda_{1}}\right)\left\{f^{\prime}(n)\left(a_{n, \lambda}-n\right)+f(n)\right\} \\
& >a_{n, \lambda}-n+n=a_{n, \lambda},
\end{aligned}
$$

which is a contradiction. Thus we conclude there exists $n_{0} \in \mathbb{N}$ such that $f_{n}\left(a_{n, \lambda}\right)=f\left(a_{n, \lambda}\right)$, and $a_{n} \geq\left(\frac{\lambda^{*}}{2 \lambda_{1}}\right) f\left(a_{n, \lambda}\right)$ for $n \geq n_{0}$. Now, by the assumption $f$, we have $C>0$ such that $f(s) \geq \frac{4 \lambda_{1}}{\lambda^{*}} s-C$ holds for any $s>0$. From this and the former estimate, we have $a_{n, \lambda} \leq\left(\frac{\lambda^{*}}{2 \lambda_{1}}\right) C$ for $n \geq n_{0}$. This implies that

$$
\left\|u_{n, \lambda}\right\|_{L^{1}(\partial \Omega)} \leq C \text { for any } n \geq n_{0} \text { and any } \lambda \in\left(\lambda^{*} / 2, \lambda^{*}\right) .
$$

At this stage, we can invoke Lemma 17 to confirm that

$$
\left\|u_{n, \lambda}\right\|_{W^{1, q}(\Omega)} \leq C\left(1 \leq q<\frac{N}{N-1}\right) \quad \text { and } \quad\left\|u_{n, \lambda}\right\|_{L^{p}(\partial \Omega)} \leq C\left(1 \leq p<\frac{N-1}{N-2}\right)
$$

for any $n \geq n_{0}$ and $\lambda \in\left(\lambda^{*} / 2, \lambda^{*}\right)$. Now, since $f_{n}(s)$ is linear for $s$ large, we have $f_{n}\left(u_{n, \lambda}\right) \in L^{p}(\partial \Omega)$ and $\left\|f_{n}\left(u_{n, \lambda}\right)\right\|_{L^{p}(\partial \Omega)} \leq C(n)$ for $1 \leq p<\frac{N-1}{N-2}$. Thus the elliptic $L^{p}$ estimate: $\left\|u_{n, \lambda}\right\|_{W^{1, \gamma}(\Omega)} \leq C\left\|f_{n}\left(u_{n, \lambda}\right)\right\|_{L^{p}(\partial \Omega)}$ where $\gamma=\frac{N p}{N-1}<$ $\frac{N}{N-2}$, and the trace Sobolev embedding: $W^{1, \gamma}(\Omega) \hookrightarrow L^{\frac{(N-1) \gamma}{N-\gamma}}(\partial \Omega)$, imply that $\left\|u_{n, \lambda}\right\|_{L^{p}(\partial \Omega)} \leq C(n)$ for any $1 \leq p<\frac{N-1}{N-3}$. We can continue this bootstrap procedure. Finally, we have $\left\|u_{n, \lambda}\right\|_{C^{2}(\bar{\Omega})} \leq C(n)$ uniformly in $\lambda \in\left(\lambda^{*} / 2, \lambda^{*}\right)$. Thus, letting $\lambda \uparrow \lambda_{n}^{*}$, we see that $u_{n, \lambda} \rightarrow u_{n}^{*}$ in $C^{1, \alpha}(\bar{\Omega})$ for some $\alpha \in(0,1)$ and $u_{n}^{*} \in C^{2}(\bar{\Omega})$ is a classical solution of

$$
\begin{cases}-\Delta u_{n}^{*}+u_{n}^{*}=0 & \text { in } \Omega, \\ \frac{\partial u_{n}^{*}}{\partial \nu}=\lambda_{n}^{*} f_{n}\left(u_{n}^{*}\right) & \text { on } \partial \Omega .\end{cases}
$$


This proves the claim.

Now, the facts that $u_{n}^{*}$ is classical and there is no classical solution of $(5.2)_{\lambda}$ for $\lambda>\lambda_{n}^{*}$, the linearized problem around $u_{n}^{*}$ must have zero eigenvalue. Thus, there exists $\varphi_{n}>0$ with $\int_{\partial \Omega} \varphi_{n} d s_{x}=1$ such that

$$
\begin{cases}-\Delta \varphi_{n}+\varphi_{n}=0 & \text { in } \Omega, \\ \frac{\partial \varphi_{n}}{\partial \nu}=\lambda_{n}^{*} f_{n}^{\prime}\left(u_{n}^{*}\right) \varphi_{n} & \text { on } \partial \Omega .\end{cases}
$$

Step 2. By letting $\lambda \uparrow \lambda_{n}^{*}$ in (5.3), we have $\left\|u_{n}^{*}\right\|_{L^{1}(\partial \Omega)} \leq C$. Also recall our normalization $\left\|\varphi_{n}\right\|_{L^{1}(\partial \Omega)}=1$. Thus by Lemma 17 , we see that there exist $w, \varphi \in L^{1}(\Omega), \varphi \geq 0$ a.e. such that

$$
\begin{array}{lll}
u_{n}^{*} \rightarrow w, & \varphi_{n} \rightarrow \varphi & \text { weakly in } W^{1, q}(\Omega), \\
u_{n}^{*} \rightarrow w, & \varphi_{n} \rightarrow \varphi & \text { strongly in } L^{p}(\partial \Omega) \text { and a.e. on } \partial \Omega
\end{array}
$$

for any $1<q<\frac{N}{N-1}$ and $1 \leq p<\frac{N-1}{N-2}$. In particular, we have $\int_{\partial \Omega} \varphi d s_{x}=1$, which implies $\varphi \not \equiv 0$ on $\partial \Omega$.

We prove that $\lambda_{n}^{*} \downarrow \lambda^{*}$ as $n \rightarrow \infty$ and $w=u^{*}$. First, we show that the weak limit $w \in W^{1, q}(\Omega)$ is a weak solution in the sense of Definition 3, when considered as $w=\left(w_{1}, w_{2}\right) \in L^{1}(\Omega) \times L^{1}(\partial \Omega)$, where $w_{1}=\left.w\right|_{\Omega}$, and $w_{2}=\left.w\right|_{\partial \Omega}$ is the usual trace of a Sobolev function $w$ on $\partial \Omega$. Indeed, put $\bar{\lambda}=\inf _{n \geq n_{0}} \lambda_{n}^{*}$. Since $\lambda_{n}^{*} \geq \lambda^{*}$ for any $n \geq n_{0}$, we have $\bar{\lambda} \geq \lambda^{*}$. For all $\zeta \in C^{2}(\bar{\Omega}), \zeta \geq 0$, we observe that

$$
\begin{aligned}
\int_{\Omega}(-\Delta \zeta+\zeta) u_{n}^{*} d x & =\lambda_{n}^{*} \int_{\partial \Omega} f_{n}\left(u_{n}^{*}\right) \zeta d s_{x}-\int_{\partial \Omega} \frac{\partial \zeta}{\partial \nu} u_{n}^{*} d s_{x} \\
& \geq \bar{\lambda} \int_{\partial \Omega} f_{n}\left(u_{n}^{*}\right) \zeta d s_{x}-\int_{\partial \Omega} \frac{\partial \zeta}{\partial \nu} u_{n}^{*} d s_{x} .
\end{aligned}
$$

Using $u_{n}^{*} \rightarrow w$ in $L^{1}(\Omega)$ on the left side hand and Fatou's lemma on the right hand side, we have

$$
\begin{aligned}
\int_{\Omega}(-\Delta \zeta+\zeta) w d x & \geq \bar{\lambda} \int_{\partial \Omega} f(w) \zeta d s_{x}-\int_{\partial \Omega} \frac{\partial \zeta}{\partial \nu} w d s_{x} \\
& \geq \lambda^{*} \int_{\partial \Omega} f(w) \zeta d s_{x}-\int_{\partial \Omega} \frac{\partial \zeta}{\partial \nu} w d s_{x}, \quad \forall \zeta \in C^{2}(\bar{\Omega}), \zeta \geq 0 .
\end{aligned}
$$

This implies also $f(w) \in L^{1}(\partial \Omega)$ if we take $\zeta \equiv 1$. Thus, we conclude that $w$ is a weak supersolution to $(1.1)_{\lambda^{*}}$ Then by Theorem 14 , we conclude that $\bar{\lambda}=\lambda^{*}$ and $w=u^{*}$. 
Step 3. Let $\varphi_{n}$ be as in (5.4). We claim that $\lambda_{n}^{*} f_{n}^{\prime}\left(u_{n}^{*}\right) \varphi_{n} \rightarrow \lambda^{*} f^{\prime}\left(u^{*}\right) \varphi$ strongly in $L^{1}(\partial \Omega)$ as $n \rightarrow \infty$.

If this claim is proved, then we pass to the limit $n \rightarrow \infty$ in the weak formulation of (5.4):

$$
\int_{\Omega}(-\Delta \zeta+\zeta) \varphi_{n} d x=\int_{\partial \Omega} \lambda_{n}^{*} f_{n}^{\prime}\left(u_{n}^{*}\right) \varphi_{n} \zeta-\frac{\partial \zeta}{\partial \nu} \varphi_{n} d s_{x}, \quad \forall \zeta \in C^{2}(\bar{\Omega}),
$$

and conclude that $\varphi$ is a weak solution of

$$
\begin{cases}-\Delta \varphi+\varphi=0 & \text { in } \Omega, \\ \frac{\partial \varphi}{\partial \nu}=\lambda^{*} f^{\prime}\left(u^{*}\right) \varphi & \text { on } \partial \Omega\end{cases}
$$

in the sense of Definition 3. Recall $\varphi \in W^{1, q}(\Omega)$ for any $1 \leq q<\frac{N}{N-1}$. Thus the proof of Theorem 16 is finished.

To prove the strong convergence $\lambda_{n}^{*} f_{n}^{\prime}\left(u_{n}^{*}\right) \varphi_{n} \rightarrow \lambda^{*} f^{\prime}\left(u^{*}\right) \varphi$ in $L^{1}(\partial \Omega)$, we invoke Vitali's Convergence Theorem. First, by (5.5), we see

$$
\lambda_{n}^{*} f_{n}^{\prime}\left(u_{n}^{*}(x)\right) \varphi_{n}(x) \rightarrow \lambda^{*} f^{\prime}\left(u^{*}(x)\right) \varphi(x) \quad \text { a.e. } x \in \partial \Omega
$$

for a subsequence. To prove the uniformly absolute continuous property of the sequence $\left\{\lambda_{n}^{*} f_{n}^{\prime}\left(u_{n}^{*}\right) \varphi_{n}\right\}_{n \in \mathbb{N}}$, let $A \subset \partial \Omega$ and $\varepsilon>0$ be given arbitrary. Convexity of $f_{n}$ implies

$$
f_{n}\left(\frac{\chi_{A}(x)}{\varepsilon}\right) \geq f_{n}\left(u_{n}^{*}(x)\right)+f_{n}^{\prime}\left(u_{n}^{*}(x)\right)\left(\frac{\chi_{A}(x)}{\varepsilon}-u_{n}^{*}(x)\right)
$$

a.e. $x \in \partial \Omega$, here $\chi_{A}$ is the characteristic function of $A$. Also from the equations satisfied by $\varphi_{n}$ and $u_{n}^{*}$,

$$
\int_{\partial \Omega} f_{n}\left(u_{n}^{*}\right) \varphi_{n} d s_{x}=\int_{\partial \Omega} f_{n}^{\prime}\left(u_{n}^{*}\right) u_{n}^{*} \varphi_{n} d s_{x}
$$


holds. Thus

$$
\begin{aligned}
\int_{\partial \Omega} f_{n}^{\prime}\left(u_{n}^{*}\right) \frac{\chi_{A}}{\varepsilon} \varphi_{n} d s_{x} & \leq \int_{\partial \Omega} f_{n}\left(\frac{\chi_{A}}{\varepsilon}\right) \varphi_{n} d s_{x}+\int_{\partial \Omega} f_{n}^{\prime}\left(u_{n}^{*}\right) u_{n}^{*} \varphi_{n} d s_{x}-\int_{\partial \Omega} f_{n}\left(u_{n}^{*}\right) \varphi_{n} d s_{x} \\
& \leq \int_{\partial \Omega} f_{n}\left(\frac{\chi_{A}}{\varepsilon}\right) \varphi_{n} d s_{x} \\
& =\int_{\partial \Omega}\left\{f_{n}\left(\frac{\chi_{A}}{\varepsilon}\right)-f(0)\right\} \varphi_{n} d s_{x}+\int_{\partial \Omega} f(0) \varphi_{n} d s_{x} \\
& \leq \int_{\partial \Omega} f\left(\frac{1}{\varepsilon}\right) \varphi_{n} \chi_{A} d s_{x}+f(0) \\
& \leq f\left(\frac{1}{\varepsilon}\right)|A|^{\frac{1}{p^{\prime}}}\left\|\varphi_{n}\right\|_{L^{p}(\partial \Omega)}+f(0) \\
& \leq C f\left(\frac{1}{\varepsilon}\right)|A|^{\frac{1}{p^{\prime}}}+f(0)
\end{aligned}
$$

for any $1 \leq p<\frac{N-1}{N-2}$, here $|A|$ denotes the $(N-1)$ dimensional measure of $A \subset \partial \Omega$. Note that

$$
\left\{f_{n}\left(\frac{\chi_{A}(x)}{\varepsilon}\right)-f(0)\right\} \varphi_{n}(x) \leq f\left(\frac{1}{\varepsilon}\right) \varphi_{n}(x) \chi_{A}(x) \quad \text { a.e. on } \partial \Omega
$$

and $\left\|\varphi_{n}\right\|_{L^{p}(\partial \Omega)} \leq C$ for some $C>0$ independent of $n$ by (5.5). Define

$$
\delta=\delta(\varepsilon)=\left(\frac{f(0)}{f\left(\frac{1}{\varepsilon}\right) C}\right)^{p^{\prime}} .
$$

Then above calculation shows that for any $\varepsilon>0$, if $A \subset \partial \Omega$ satisfies that $|A|<\delta(\varepsilon)$, we obtain $\int_{A} f_{n}^{\prime}\left(u_{n}^{*}\right) \varphi_{n} d s_{x} \leq 2 f(0) \varepsilon$. Thus the uniform absolutely continuity of the sequence $\left\{\lambda_{n}^{*} f_{n}^{\prime}\left(u_{n}^{*}\right) \varphi_{n}\right\}_{n \in \mathbb{N}}$ is confirmed. Also if we take $E \subset \partial \Omega$ such that $|\partial \Omega \backslash E|<\delta$ where $\delta$ is as above, we obtain the uniform integrability of $\left\{\lambda_{n}^{*} f_{n}^{\prime}\left(u_{n}^{*}\right) \varphi_{n}\right\}_{n \in \mathbb{N}}$ : for any $\varepsilon>0$, there exists $E \subset \partial \Omega$ such that $\int_{\partial \Omega \backslash E} \lambda_{n}^{*} f_{n}^{\prime}\left(u_{n}^{*}\right) \varphi_{n} d s_{x} \leq C \varepsilon$. Therefore, Vitali's Convergence Theorem assures the claim.

Acknowledgement. Part of this work was supported by JSPS Grant-inAid for Scientic Research (B), No. 23340038, and JSPS Grant-in-Aid for Challenging Exploratory Research, No. 24654043. 


\section{References}

[1] S. Agmon, A. Douglis, and L. Nirenberg: Estimates near the boundary for solutions of elliptic partial differential equations satisfying general boundary conditions, I, Comm. Pure Appl. Math., 12, 623-727, (1959)

[2] S. Agmon, A. Douglis, and L. Nirenberg: Estimates near the boundary for solutions of elliptic partial differential equations satisfying general boundary conditions, II, Comm. Pure Appl. Math., 17, 35-92, (1964)

[3] H. Brezis, T. Cazenave, Y. Martel and A. Ramiandorisoa: Blow up for $u_{t}-\Delta u=g(u)$ revisited, Adv. Differential Equations. 1, 73-90, (1996)

[4] E. Berchio, F. Gazzola, and D. Pierotti: Gelfand type elliptic problems under Steklov boundary conditions, Ann. Inst. H. Poincaré Anal. Non Linéaire 27, 315-335, (2010)

[5] H. Brezis, and W. A. Strauss: Semi-linear elliptic equations in $L^{1}$, J. Math. Soc. Japan., 25, 565-590, (1973)

[6] H. Brezis, and J. L. Vázquez: Blow-up solutions of some nonlinear elliptic problems, Rev. Mat. Univ. Compl. Madrid, 10, 443-469, (1997)

[7] X. Cabré, and Y. Martel: Weak eigenfunctions for the linearization of extremal elliptic problems, J. Funct. Anal. 156, 30-56, (1998)

[8] X. Cabré: Regularity of minimizers of semilinear elliptic problems up to dimension 4, Comm. Pure Appl. Math. 63, 1362-1380, (2010)

[9] M.G. Crandall, and R.H. Rabinowitz: Some continuation and variational methods for positive solutions of nonlinear elliptic eigenvalue problems, Arch. Rational Mech. Anal. 58, 207-218, (1975)

[10] J. Dávila: Singular solutions of semi-linear elliptic problems, Handbook of differential equations: stationary partial differential equations. Vol. VI, 83-176, (2008)

[11] J. Dávila, L. Dupaigne, and M. Montenegro: The extremal solution of a boundary reaction problem, Commun. Pure Appl. Anal. 7, 795-817, (2008) 
[12] L. Dupaigne: Stable Solutions of Elliptic Partial Differential Equations, Monographs and Surveys in Pure and Applied Mathematics 143, Chapman \& Hall/CRC Press, xiv+321 pp. (2011)

[13] Y. Martel: Uniqueness of weak extremal solutions of nonlinear elliptic problems, Houston J. Math. 23, 161-168, (1997)

[14] F. Mignot, and J.P. Puel: Sur une classe de problèmes non linéaires avec nonlinéairité positive, croissante, convexe, Comm. Partial Differential Equations. 5, 791-836, (1980)

[15] G. Nedev: Regularity of the extremal solution of semilinear elliptic equations, C. R. Acad. Sci. Paris Sér.I Math. 330, 997-1002, (2000)

[16] P. Quittner, and W. Reichel: Very weak solutions to elliptic equations with nonlinear Neumann boundary conditions, Calc. Var. 32, 429-452, (2008)

[17] J. D. Rossi: Elliptic problems with nonlinear boundary conditions and the Sobolev trace theorem, Handbook of differential equations: stationary partial differential equations. Vol. II, 311-406, (2005)

[18] S. Villegas: Boundedness of extremal solutions in dimension 4, Adv. Math. 235, 126-133, (2013) 